\title{
eNeuro
}

Research Article: New Research / Cognition and Behavior

\section{Phase-synchronized stimulus presentation augments contingency knowledge and affective evaluation in a fear conditioning task}

https://doi.org/10.1523/ENEURO.0538-20.2021

Cite as: eNeuro 2021; 10.1523/ENEURO.0538-20.2021

Received: 4 December 2020

Revised: 15 October 2021

Accepted: 26 October 2021

This Early Release article has been peer-reviewed and accepted, but has not been through the composition and copyediting processes. The final version may differ slightly in style or formatting and will contain links to any extended data.

Alerts: Sign up at www.eneuro.org/alerts to receive customized email alerts when the fully formatted version of this article is published.

Copyright (c) 2021 Plog et al.

This is an open-access article distributed under the terms of the Creative Commons Attribution 4.0 International license, which permits unrestricted use, distribution and reproduction in any medium provided that the original work is properly attributed. 
1 Title: Phase-synchronized stimulus presentation augments contingency knowledge

2 and affective evaluation in a fear conditioning task

3

4 Abbreviated Title: Phase-synchronization in fear conditioning

5 Author names and affiliations: Elena Plog ${ }^{1 *}$, Martin I. Antov ${ }^{1 *}$, Philipp Bierwirth ${ }^{1}$, Andreas

6 Keil $^{2}$, and Ursula Stockhorst ${ }^{1}$

$7 \quad{ }^{1}$ Insitute of Psychology, Experimental Psychology II and Biological Psychology, University of

8 Osnabrück, D-49074 Osnabrück, Germany (E. Plog, M. I. Antov, P. Bierwirth, and U.

9 Stockhorst)

10 'Department of Psychology and Center for the Study of Emotion and Attention, University of 11 Florida, Gainesville, Florida, 32611, USA

Authors Contributions: E. Plog designed research, performed research, analyzed data, wrote the paper (first draft); M.I. Antov designed research, performed research, analyzed data and wrote the paper (first draft); P. Bierwirth analyzed data, A. Keil, contributed analytic tools, wrote the paper, $U$. Stockhorst designed research and wrote the paper

* These two authors contributed equally.

Correspondence should be addressed to: Elena Plog, D-49074 Osnabrück, E-Mail:

18 eplog@uni-osnabrueck.de

19 Number of Figures: 8

20 Number of Tables: 1

21 Number of Multimedia: 0

22 Number of extended data: 11 figures

23 Number of words for Abstract: 249 words

24 Number of words for Significance Statement: 89

25 Number of words for Introduction: 748

26 Number of words for Discussion: 2749

Acknowledgements: We thank Jelena Gildehaus and Tabea Rasche for help with the recruitment of participants and data collection

Conflict of Interest: The authors declare no competing financial interests.

30 Funding sources: The study was supported by the research profile line "Cognition" of the 31 University of Osnabrück, Germany 
Phase-synchronized stimulus presentation augments contingency knowledge and affective evaluation in a fear conditioning task

\section{Abstract}

Memory often combines information from different sensory modalities. Animal studies show that synchronized neuronal activity in the theta band $(4-8 \mathrm{~Hz})$ binds multimodal associations. Studies with human participants have likewise established that theta-phase synchronization augments the formation of declarative video-tone pair memories. Another form of associative learning, classical fear conditioning, models non-declarative, emotional memory - with distinct neuronal mechanisms. Typical fear conditioning tasks pair a conditioned stimulus (CS) in one modality with an aversive unconditioned stimulus (US) in another. The present study examines the effects of CS-US synchronization in the theta band on fear memory formation in humans.

In a fear generalization procedure, we paired one of five visual gratings of varying orientation (CS) with an aversive auditory US. We modulated the luminance of the CS and the volume of the US at a rate of $4 \mathrm{~Hz}$. To manipulate the synchrony between visual and auditory input during fear acquisition, one group $(\mathrm{N}=20)$ received synchronous CS-US pairing, whereas the control group $(N=20)$ received the CS-US pairs out-of-phase.

Phase synchronization improved CS-US contingency knowledge and facilitated CS discrimination in terms of rated valence and arousal, resulting in narrower generalization across the CS gratings compared to the out-of-phase group. In contrast, synchronization did not amplify conditioned responding in physiological arousal (skin conductance) and visuocortical engagement (steady-state visually evoked potentials) during acquisition, although both measures demonstrated tuning towards the CS+. Together, these data support a causal role of theta-phase synchronization in affective evaluation and contingency report during fear acquisition. 
59 Due to methodological limitations, examining the causal role of oscillatory synchrony in 60 association formation has been challenging so far. Using repetitive, rhythmic sensory stimulation in a memory-related $4 \mathrm{~Hz}$ frequency, we examined the role of phase

62 synchronization in fear conditioning. While synchronization improved the contingency knowledge and affective evaluation, physiological arousal and visuocortical activity were unaffected by the phase-modulation. Our results represent an initial step towards establishing the causal effects of theta phase synchronization in associative fear learning, thus improving our understanding of the neurophysiological mechanisms of fear-memory encoding.

\section{Introduction}

Phase-synchronization of brain oscillations has been proposed as a mechanism supporting neuronal communication and plasticity (Fell \& Axmacher, 2011). A theoretical perspective holds that the ongoing oscillatory phase reflects the excitability of a neural population and therefore determines a window for successful long-term-potentiation (LTP), a cellular process underlying learning and plasticity (Lynch, 2004). Research in rodents has shown that the induction of LTP or long-term depression (LTD) critically depends on oscillatory phases and the stimulation or recording site: While LTP was induced in behaving rats when the hippocampal CA1 was stimulated at the oscillatory peak, long-term depression (LTD) resulted from stimulation at the trough (Hyman et al., 2003). Using trace eyeblink conditioning in rabbits and recordings in the hippocampal fissure, CS-presentation in the trough induced phase-locked, regular (theta) oscillations that were in turn associated with better learning whereas CS-presentation to the peak impaired regularity and learning (Nokia et al., 2015). Note, that the theta phase reverses between the hippocampal fissure and the

82 CA1 region. Since LTP requires precise timing between pre-and postsynaptic activation in the millisecond range (Markram et al., 1997), orchestrating activity by phase synchronization of neuronal oscillations is a potential mechanism supporting LTP. Among other oscillatory 
phenomena, oscillations in the theta range $(4-8 \mathrm{~Hz}$ in primates, $4-12 \mathrm{~Hz}$ in rodents) and their synchronization between memory-related brain sites are linked to memory performance (Headley \& Paré, 2017). Rodent research (Benchenane et al., 2010; Place et al., 2016) and human EEG-studies (Summerfield \& Mangels, 2005; Weiss \& Rappelsberger, 2000) found increased theta synchronization between brain regions during different episodic memory tasks, suggesting that theta-synchronization facilitates communication (Fell \& Axmacher, 2011).

Intriguingly, studies in humans have causally linked theta-phase synchronization to episodic associative memory. Repetitive, rhythmic sensory stimulation eliciting steady-state-evoked potentials (Clouter et al., 2017; Wang et al., 2018), enables experimental control over response frequency in a sensory region, and corresponding phase synchrony between regions (HansImayr et al., 2019; Herrmann et al., 2016; Thut et al., 2011). Synchronizing the oscillatory phase evoked by periodically modulated visual and auditory stimuli facilitated the encoding of an episodic audio-visual memory (Clouter et al., 2017; Wang et al., 2018), suggesting theta-phase synchronization as a mechanism for binding multisensory episodic memories. The synchronized input is assumed to increase temporally organized neuronal firing, which in turn may result in LTP (Buzsáki, 2002; Fries, 2015).

Although LTP is best understood in the hippocampus, its associative and synapse-specific properties make it a potential mechanism for plasticity in other regions (Bliss et al., 2018; Maren \& Fanselow, 1995; Orsini \& Maren, 2012). E.g., fear conditioning, a paradigm of associative emotional memory, involves associative plasticity within the lateral nucleus of the amygdala (Kim \& Cho, 2017), but also in other structures processing the conditioned (CS) and unconditioned (US) stimuli (Herry \& Johansen, 2014). Sensory information of both stimuli (typically different modalities) converge onto the same neuronal populations in the lateral amygdala (LA; Romanski et al., 1993). Activating weak CS synapses in temporal proximity to strong US synapses initiates a cascade of cellular reorganization, strengthening CS synapses and enabling the CS to elicit fear responses (Blair et al., 2001; Orsini \& Maren, 2012). Importantly, theta synchronization between medial prefrontal cortex, amygdala, and 
113 hippocampus plays a role during fear conditioning (Karalis et al., 2016; Seidenbecher et al.,

114 2003; Taub et al., 2018; Zheng et al., 2019; for reviews see Bocchio et al., 2017; Çalişkan \&

115 Stork, 2018). However, its causal role in forming CS-US associations, is unknown.

116 The current study asked if synchronized sensory input helps the formation of a multisensory

117 CS-US association in aversive learning. Using rhythmic external stimulation (cf., Clouter et 118 al., 2017; Wang et al., 2018), we utilized "in-phase" vs. "out-of-phase" presentation of the 119 visual CS and auditory US, in a 2-day fear conditioning using a generalization paradigm (5 120 similar CS). We hypothesized that theta-band (4 Hz) synchronization of two distinct sensory 121 systems promotes the CS-US association. Specifically, it was expected that in-phase 122 presentation facilitates fear acquisition, whereas out-of-phase presentation prompts poor fear 123 conditioning. Synchronizing the multisensory input is expected to orchestrate neuronal 124 activity in the sensory cortices (so-called entrainment). If synchronization in the theta range 125 provides a window for successful LTP, it should optimize conditions for synchronous afferent 126 signals reaching further structures within the fear network, especially the lateral amygdala 127 (LeDoux, 2000; Romanski et al., 1993). To assess different response systems in human fear 128 conditioning, we measured conditioned responses in physiological arousal, affective 129 evaluation of arousal and valence, contingency knowledge of CS and US, and visuocortical 130 engagement. 
134 The final sample comprised 40 healthy, right-handed students of the University of Osnabrück 135 between 19 and 30 years $(M=22.2, S E M=0.35 ; 20$ men, 20 women). To control for sex-

136 hormone fluctuations, female participants were only included if they used monophasic oral 137 contraceptives (pill) and were examined between the $6^{\text {th }}$ and $20^{\text {th }}$ day of pill-intake (i.e., in the 138 pill-on phase). Participants were screened via self-report questionnaire and a structured interview for inclusion and exclusion criteria in a screening session that was always

140 conducted on a different day than the actual main experiment. Students with acute or chronic physical and/or psychiatric disorders (e.g., migraine, epilepsy, cardiovascular diseases and 142 phobias) were not eligible. Further exclusion criteria encompassed hearing and/or 143 uncorrected vision impairments, tinnitus, acute medication, drug abuse, average alcohol 144 consumption exceeding $20 \mathrm{~g}$ or $40 \mathrm{~g}$ ethanol / day (for women and men, respectively), and 145 smoking of $>5$ cigarettes per day. Volunteers were screened for posttraumatic stress 146 disorder (PTSD) using a translated version of the Posttraumatic Stress Diagnostic Scale 147 (Foa, 2000; Steil \& Ehlers, 2000) and excluded if they met the DSM-IV criteria for PTSD. 148 From 64 volunteers (34 women), 46 (25 women) were eligible to participate, 3 women did not 149 appear to the appointment and 3 ( 2 women) discontinued the main experiment due to the 150 aversive nature of the conditioning paradigm, leading to our final sample of 40 participants. 151 Within the female and male subsample, participants were randomly assigned to one of two 152 groups: In-phase or out-of-phase, with the same number of men and women in each group 153 (in-phase: 10 women; out-of-phase: 10 women).

154 The study was approved by the ethics committee of the University of Osnabrück and 155 conducted in accordance with the Declaration of Helsinki guidelines. Written informed 156 consent was obtained from all participants after adequate understanding of the explained 157 procedures. Each participant was free to choose between participation credits (4 credits) or a 
158

159

160

161

162

163

164

165

166

167

168

169

170

171

172

173

174

175

176

177

178

179

180

181

182

183

184

corresponding amount of money (32 €) for finishing screening and day 1 and 2 of our conditioning procedure.

\section{Experimental design and stimuli}

We used a 2-day differential fear conditioning procedure, including habituation, acquisition, immediate extinction on day 1 , and a 24-h delayed recall on day 2 (Figure 1B). Our study comprised a $5 \times 2$ mixed factorial design within each learning phase, with 5 CS-orientations of the below characterized Gabor-gratings $\left(25^{\circ}, 35^{\circ}, 45^{\circ}, 55^{\circ}\right.$, and $\left.65^{\circ}\right)$ as the within-subject factor, and synchronization (in-phase, i.e., $0^{\circ}$ phase shift $v s$. out-of-phase, i.e., $90^{\circ}, 180^{\circ}$, and $270^{\circ}$ phase shift) as experimental between-subject factor.

Five high-contrast, black-and-white Gabor gratings (i.e., a sinusoid grating filtered with a Gauss-function) with a low spatial frequency served as the visual CS. The five CS differed only in orientation $\left(25^{\circ}, 35^{\circ}, 45^{\circ}, 55^{\circ}\right.$, and $65^{\circ}$, relative to vertical $0^{\circ}$, Figure $\left.1 A\right)$. The CS were presented for $5 \mathrm{~s}$ centrally on a dark grey background (100\% black setting on the monitor). During the presentation the experimental chamber was only lit by the CS on the screen. Technical failure forced us to exchange the monitor from a 19" (Acer P911) to a 17" (Sony, CPD-E220E) cathode ray tube (CRT) after examining the first 12 participants. We matched stimulus properties as closely as possible with the new monitor. The relevant parameters were comparable: i.e., $85 \mathrm{~Hz}$ refresh rate, low spatial frequency (0.96 vs. 0.81 cycles/degree), large central CS presentation (5.70 vs. $5.73^{\circ}$ visual angle), high contrast (96 $\%$ Michelson for both monitors).

As the US, we employed a 2-s, broadband white noise $(20 \mathrm{~Hz}-22 \mathrm{kHz}, 44100 \mathrm{Bit} / \mathrm{s}, 16$ Bits/sample), presented binaurally at a maximum of $96.5 \mathrm{~dB}(\mathrm{~A})$ over two loudspeakers positioned $0.7 \mathrm{~m}$ left and right behind the participant. For an additional unimodal audio task (at the end of session on day 2) we presented the same white noise for $4 \mathrm{~s}$ at a non-aversive sound-pressure level with a maximum of $70.4 \mathrm{~dB}(\mathrm{~A})$.

The intensity of the visual CS, the aversive auditory US and the non-aversive auditory noise (unimodal task) was modulated at $4 \mathrm{~Hz}$ (cf., Clouter et al., 2017). The luminance of the visual 
CS was sinusoidally modulated in $4 \mathrm{~Hz}$, where luminance changed at each screen refresh, resulting in 21 steps per cycle (0-100 \% luminance). The amplitude of the auditory signal was sinusoidally modulated (0-100 \%) in $4 \mathrm{~Hz}$ by multiplying the signal vector with a $4 \mathrm{~Hz}$ sine wave at the native $44.1 \mathrm{kHz}$ audio sampling rate. Presentation of each $4-\mathrm{Hz}$ modulated stimulus (auditory and visual) always started at $0 \%$ intensity, increasing to $100 \%$ in the first half cycle.

\section{--Insert Figure 1 here-}

\section{Conditioning procedure}

Our procedure included habituation, acquisition, and immediate extinction on day 1 , as well as a $24-\mathrm{h}$ delayed recall on day 2 (Figure 1B). During habituation, each $5-\mathrm{s}, 4-\mathrm{Hz}$ modulated CS was presented 12 times in pseudorandom order. Before acquisition, participants were instructed that only one of the 5 CS orientations will be followed by an aversively loud noise, without specifying which orientation. During the acquisition phase, each $4-\mathrm{Hz}$ modulated CS was again presented 12 times. However, the $45^{\circ} \mathrm{CS}(\mathrm{CS}+)$ was always paired (12 times) with the 2-s, 4-Hz modulated aversive noise US (reinforcement rate: 100\%), while the other orientations were not $\left(25^{\circ}, 35^{\circ}, 55^{\circ}\right.$, and $65^{\circ}$ gratings as CS-, Figure $\left.1 B\right)$. Previous work has used this same generalization paradigm, with $45^{\circ}$ gratings serving as $\mathrm{CS}+$, while also establishing that there are no systematic pre-conditioning differences between different grating orientations on the measures used here (McTeague et al., 2015). Previous work has also demonstrated that conditioning is seen across orientations and with counterbalancing (e.g., Moratti \& Keil, 2005). Together, to facilitate interpretation and comparison with prior work, this led us to adopt a fixed contingency between $45^{\circ}$ and the US. Each CS presentation was prolonged for the duration of the US, adding $2 \mathrm{~s}$ (i.e., 7-s duration for the $\mathrm{CS}+$ and the CS- gratings during acquisition). For the $45^{\circ} \mathrm{CS}+$, the last $2 \mathrm{~s}$ of visual CS overlapped with the auditory US presentation. Since retinal phototransduction was shown to be slower than auditory transduction ( $\sim 50 \mathrm{~ms}$ for visual stimuli vs. $10 \mathrm{~ms}$ for auditory stimuli; King \& Palmer, 1985; Lennie, 1981), the onset of the auditory US had a 40 ms delay relative 
213 to the CS onset (cf. Clouter et al., 2017). The slower transduction of visual stimuli is also in

214 line with recordings in the amygdala after visual vs. auditory stimulation. The earliest activity

215 occurred between 40 and $80 \mathrm{~ms}$ (up to $316 \mathrm{~ms}$, depending on the pathway to the amygdala

216 that differs in length) after visual stimulation (Luo et al., 2010; McFadyen et al., 2017;

217 Silverstein \& Ingvar, 2015 for a review). In contrast, auditory information was recorded as

218 early as $10-40 \mathrm{~ms}$ in single-units of anesthetized as well as freely-moving rats (Quirk et al.,

219 1995; Romanski et al., 1993). This temporal difference in processing from receptors to the

220 afferent neurons in the CNS has to be considered when entraining the brain with

221 multisensory information in a synchronous way. Thus, adjusting for a temporal delay in one

222 modality is necessary to achieve theta synchronization of visual CS and auditory US in the

223 sensory cortices and facilitating synchrony of both input on the LA. CS presentation followed

224 one of two random sequences (Extended data Figure 1-1), with trial order constrained to not

225 allow more than two consecutive CS of the same orientation. Additionally, the acquisition

226 phase started with a booster sequence, where 5 of the first 7 trials were CS+US pairings.

227 The following immediate extinction, and the 24-h delayed recall phase comprised only CS

228 presentations (12 times each, no US), as in the habituation phase. The aversive US was not

229 presented at any point besides the 12 CS+US pairings in the acquisition phase. However,

230 neither before immediate extinction learning, nor before $24-\mathrm{h}$ delayed recall, participants

231 were informed that no US will occur in the following stimulation phase.

232 Between the end of one CS presentation and the beginning of next one, a black screen was

233 shown for 3 to $5 \mathrm{~s}$ (random from a uniform distribution) during learning phases (1.5 to $3 \mathrm{~s}$

234 during the unimodal audio task), followed by $1.5 \mathrm{~s}$ white fixation cross at the center of the

235 screen, resulting in an inter-trial-interval (ITI) between 4.5 and $6.5 \mathrm{~s}$.

236 In accordance with Clouter et al. (2017), the 12 pairings of the 4-Hz modulated CS+ and US

237 were realized with either $0^{\circ}$ (i.e., $\left.0 \mathrm{~ms}\right)$ phase-shift (in-phase group), or in a $90^{\circ}(62.5 \mathrm{~ms})$,

$238180^{\circ}(125 \mathrm{~ms})$, and $270^{\circ}(187.5 \mathrm{~ms})$ shift for 4 USs each (out-of-phase group, Figure $1 C$ ).

239 Accounting for the 40-ms delay between rapid auditory and later visual processing times, 
240

241

242

243

244

245

246

247

248

249

250

251

252

253

254

255

256

257

258

259

260

261

262

263

264

265

266

input with a phase-lag of $0^{\circ}$ causes phase-synchronized cortical activity in the visual and auditory cortex (Clouter et al., 2017). This synchronized activity at the primary cortices is expected to increase the synchronized afferent signals reaching the amygdala, where CS-US convergence occurs, hence supporting associative plasticity in the lateral amygdala (Blair et al., 2001; Bocchio et al., 2017). In contrast, phase lags between $90^{\circ}$ and $270^{\circ}$, i.e., timing shifts of $62.5-187.5 \mathrm{~ms}$, should result in a suboptimal level of excitability and therefore decrease the likelihood of synaptic changes.

The experiment was conducted in an electromagnetically shielded and sound-attenuated experimental chamber, where participants were seated in a comfortable chair positioned centrally in front of the monitor. The experiment including all stimuli was created in MATLAB (2019b, RRID:SCR_001622) using the Psychophysics Toolbox (RRID:SCR_002881; (Brainard, 1997; Kleiner et al., 2007).

\section{Sensory stimulation validation}

To validate the temporal fidelity of the stimulation, we analyzed data from two sources: a photo diode (Brain Products, Photo Sensor) attached to the participants' monitor, and a microphone (built into a BrainVision StimTrak) positioned in front of the participant's speakers. Both signals were recorded at $1000 \mathrm{~Hz}$ in BrainVision Recorder. The photo diode was placed over the upper right corner of the CRT monitor where a smaller version of the Gabor gratings appeared during the same monitor refresh cycle (and far outside of CS presentation area) in the same sinusoidal luminance modulation as the original CS-gratings. Pilot studies using photo sensors at both the upper corner (small test Gabor) and central screen (actual CS grating) showed excellent synchrony of both stimuli. The onset of the central grating was consistently 0.5 refresh cycles after the onset of the miniature grating in the top left corner, i.e. $5.9 \mathrm{~ms}$. Using BrainVision Analyzer, data from the photo sensor and the microphone were segmented from -320 to 2500 ms relative to the onset of an US (i.e., 12 segments per subject), and visually inspected for artifacts. A subset of segments was 
excluded, as microphone data were corrupted or missing due to failure of the StimTrack batteries (in 5 out of 40 participants). For visualization, the remaining data were exported to MATLAB and rescaled from -pi to +pi. Further analysis was computed over 7.02 cycles of microphone and video data (1755 ms), disregarding the last cycle of audio stimulation. Video data from $-40 \mathrm{~ms}$ to $+1715 \mathrm{~ms}$ relative to US onset were used. In contrast, microphone data from 0 to $1755 \mathrm{~ms}$ entered analysis. This effectively shifts video data $40 \mathrm{~ms}$ forward in time, to account for the $40 \mathrm{~ms}$ time shift programmed into the stimulus presentation. Microphone data were first rectified (square root of the signal squared). Both, photo sensor and microphone, channels were band-pass filtered between 3 and $5 \mathrm{~Hz}$, using the bandpass function of MATLAB's Signal Processing Toolbox with an IIR filter (60 dB attenuation at the edge frequencies) and a steepness of .95. Instantaneous phase information at $4 \mathrm{~Hz}$ for audio and video signal was extracted from the imaginary part of the analytic signal after a Hilbert transform.

This analysis also demonstrates that there was very little variability in the timing of sensory stimulation within a trial, as well as between trials and between participants of one group.

Dependent variables

ssVEPs (via EEG), skin conductance responses (SCRs), and subjective ratings served as dependent outcomes. Further, horizontal and vertical eye movements were recorded by electrooculography (EOG) with a bipolar BrainAmpExG amplifier (BrainProducts) to detect and eliminate artifacts in the EEG-recordings.

EEG-parameters

EEG-recording and pre-processing

292

A 64-channel EEG was recorded on both days with two 32-channel BrainAmp DC amplifiers with a resolution of $0.1 \mu \mathrm{V}$ (Brain Products). The 64 active electrodes $(\mathrm{Ag} / \mathrm{AgCl}$, actiCAP, 
Brain Products) were filled with electrolyte gel (Super-Visc $10 \% \mathrm{NaCl}$, EasyCap) and positioned according to the extended international 10-20 system. Efforts were made to keep impedances below $5 \mathrm{k} \Omega$ (manufacturer's recommendation $<25 \mathrm{k} \Omega$ ). FCz served as recording reference and AFz as ground. A sampling rate of $1000 \mathrm{~Hz}$ and a high-pass filter at $0.016 \mathrm{~Hz}$ was used. In addition to the EEG, electrooculography was recorded with $4 \mathrm{Ag} / \mathrm{AgCl}$ electrodes $(\varnothing 4 \mathrm{~mm})$ to control for eye movements: Two electrodes were placed on the lateral canthus of each eye for horizontal and two electrodes were placed infra- and supraorbital, in line with pupil of the right eye, for vertical movements. An electrode on the forehead was attached as ground.

Offline pre-processing was done with Brain Vision Analyzer 2 software (version: 2.1.2.327). Raw data was band-pass filtered between $1 \mathrm{~Hz}$ and $100 \mathrm{~Hz}$, using Butterworth (zero phase shift) filters with a $3 \mathrm{~dB}$ low cutoff at $1 \mathrm{~Hz}$ (time constant: 0.1592 , order 8 ) and a $3 \mathrm{~dB}$ high cutoff at $100 \mathrm{~Hz}$ (order 4). Additional 50 and $100 \mathrm{~Hz}(1 \mathrm{~Hz}$ bandwidth, order 4) notch filters were applied to eliminate line noise. Data were segmented from -1250 to $7500 \mathrm{~ms}$ relative to a CS onset and an ocular correction independent component analysis (ICA), as implemented in BrainVision Analyzer, was applied. After visual inspection of the resulting factors and factor topographies, factors related to horizontal and vertical eye movements, blinks, as well as strong cardiac or muscular artefacts were removed from the reconstructed data. ICAcorrected data were re-referenced to an average reference, and the recording reference was re-included in the data as a $65^{\text {th }}$ channel, at position FCz. The segments were cut to an interval between -1000 to $5000 \mathrm{~ms}$ relative to CS onset. With this segmentation, the US intervals were excluded from further analyses, to avoid contamination of our EEG-data. After another visual inspection, we rejected segments with remaining artefacts. On average, 3.93 segments were rejected per participant (0-15 rejected out of 240 segments for each 318 participant). Data were down-sampled to $512 \mathrm{~Hz}$, in accordance with Clouter et al. (2017) 319 and exported to MATLAB (Mathworks, RRID:SCR_00162). To increase spatial specificity, 320 reduce volume conduction effects and to obtain reference-free data, we conducted a scalp current source density (CSD) transform (Junghöfer et al., 1997). The CSD values (as 
estimates of cortical surface potentials) are represented on a sphere, approximating a cortical surface. For scalp-level analyses and topographical mapping, the CSD was projected back onto the original electrode space. Analyses were performed on CSD-transformed data, and CSD data are shown throughout the figures.

\section{Validation of visual and auditory entrainment (unimodal)}

To validate the visual cortical entrainment at $4 \mathrm{~Hz}$, we first averaged CSD-transformed data over all habituation trials at each sensor of a participant in the time domain (disregarding CSorientations, i.e. averaging $5 \times 12$ trials per subject). Habituation trials were not only strictly unimodal visual stimulation but precede any pairing of the CS with the aversive US. To avoid early ERP entering the frequency domain analysis, Fourier transform was applied on data from 750 to $5000 \mathrm{~ms}$ (i.e., containing 17 cycles of $4 \mathrm{~Hz}$ ), post CS-onset. These data were windowed with a cosine-square window (20-point rise/fall) and subjected to a discrete Fourier transform (MATLAB) with a frequency resolution of $0.24 \mathrm{~Hz}$. We extracted the absolute values of the Fourier coefficients at $4 \mathrm{~Hz}$ and transformed the resulting power values to signal-to-noise ratios (SNRs), using the average of 5 frequency bins under and 4 frequency bins above $4 \mathrm{~Hz}$.

To validate auditory entrainment, we used the unimodal audio task at the end of day 2 , as it reflected $4-\mathrm{Hz}$ unimodal auditory stimulation, without concurrent visual stimulation. EEG-data from the audio-only task were subjected to the same pre-processing pipeline as CS-related data. As for visual unimodal data above, CSD-data were segmented (here from -1000 to $4100 \mathrm{~ms}$, relative to audio stimulus onset) and averaged per participant and sensor over the 75 audio-only trials. Fourier transform was applied on windowed data (cosine-square, 20point rise/fall) starting from 500 to $4000 \mathrm{~ms}$ after audio onset (i.e., containing 14 cycles of 4 $\mathrm{Hz}$ ), resulting in a frequency resolution of $0.29 \mathrm{~Hz}$. Like for the visual stimulation, we converted the power at $4 \mathrm{~Hz}$ to SNRs, using the average of the five frequency bins below and four above the frequency of interest as noise estimates. 
Figure 3 shows the scalp distribution of the $4-\mathrm{Hz}$ SNR averaged over participants $(N=40)$,

350

for the visual (Figure $3 \boldsymbol{A}$ ) $4-\mathrm{Hz}$ stimulation and the auditory $4-\mathrm{Hz}$ stimulation (Figure $3 \boldsymbol{B}$ ). The average topographies are consistent with a typical visual and auditory steady-state evoked potential at $4 \mathrm{~Hz}$, respectively. Specifically, the relatively low driving frequency of 4 $\mathrm{Hz}$ has traditionally been shown to prompt larger spread of the ssVEP topography, reflective of longer individual stimulation cycles, which allow spreading across the visual hierarchy (e.g., Skrandies, 2007). However, the topographies of the 4-Hz EEG-signal showed some variation between subjects. Therefore, for subsequent analyses in the frequency domain, including single-trial analyses of CS-related activity, we selected the individual 6 sensors for each participant showing the highest SNR at $4 \mathrm{~Hz}$.

--Insert Figure 3 here--

\section{ssVEP single-trial analysis}

We conducted a single-trial analysis to be able to show the temporal evolution of visual cortical engagement over the course of learning trials. For single-trial analysis, we used data segments between -1000 to $5000 \mathrm{~ms}$, relative to CS-onset as 0 (in sample points at $512 \mathrm{~Hz}$ sample rate, this is 1 to 3072 sample points with zero being sample point 512 ). First, we sampled it up from 512 to $1536 \mathrm{~Hz}$. Up-sampling the data ensured an integer number of sampling points per one cycle for the $4 \mathrm{~Hz}$ as well as its harmonics (up to $16 \mathrm{~Hz}$ ). At 1536 $\mathrm{Hz}$, one cycle of the driving frequency $(4 \mathrm{~Hz})$ is 384 samples (instead of 128 at $512 \mathrm{~Hz}$ ). By subtracting the mean of the $1000 \mathrm{~ms}$ pre-stimulus interval, the data were baseline-corrected. The power extraction of single trials was based on the analysis window between 750 and $5000 \mathrm{~ms}$ (relative to $0 \mathrm{~ms}=\mathrm{CS}$-onset). Over this analysis-window, a moving average procedure was conducted. We obtained averages by shifting a window with a length of 4 cycles of the frequency of interest (i.e., $4 \mathrm{~Hz}$ ) across the detrended data segments in steps of one cycle and averaging the contents of the window with each step (12 steps, last 4-cycle step starting at $3000 \mathrm{~ms}$ after CS-onset). We then transformed the single-trial estimates from the time into the frequency domain using discrete Fourier transform (DFT) and extracted the 
power at the driving frequency as the absolute of the Fourier coefficients, normalized by the

378 length of FFT (here, 1536 sample points).

379 Interindividual variance in response strength and pre-experimental bias was corrected by 380 calculating a habituation ratio for each CS (via division by habituation mean over all 60 trials 381 of each participant, disregarding the different CS-orientation), with values larger than 1 382 describing an enhancement and lower than 1 describing a decrease of ssVEP-power 383 compared to habituation. In addition, single-trial data were smoothed with a moving average along the 12 trials (5-point symmetrical, shrinking at the endpoints) within each learning phase and CS-orientation (5 orientations $\times 4$ learning phases with 12 trials each). For plots, showing the temporal evolution of ssVEP over trials we pooled data over sensors as the average of the individually defined six maximal SNR sensors for each participant. The individual sensors entering this 6 -sensor cluster were defined as the 6 sensors showing the highest SNR at $4 \mathrm{~Hz}$ during habituation trials for a participant (see above, Validation of visual and auditory entrainment [unimodal]). Of note: while single-trial data is interesting and informative, we have no prior evidence allowing us to formulate specific hypotheses about group differences (in-phase vs. out-of-phase) in the temporal dynamics of ssVEP. Therefore, these data were averaged over all trials of a learning phase before statistical testing for group effects.

Skin conductance responses (SCR) and electrocardiography (ECG), and blood pressure

Besides SsVEP-power tunings towards specific CS-gratings, we used SCRs as a common measure of learning-induced changes in physiological arousal to the CS. However, as our lab is configurated for stress-associated questions by default, we also recorded ECG and BP as control parameters only. ECG and BP will not be reported in the result section. We used a coupler to record SCRs with a sampling rate of $1000 \mathrm{~Hz}$ and a resolution of $0.0061 \mu \mathrm{S}$. We 
attached two $\varnothing 10 \mathrm{~mm}$ (inner diameter) electrodes, filled with $0.05 \% \mathrm{NaCl}$ paste (TD-246) on the thenar and hypothenar of the left hand (non-dominant) of each participant (Boucsein et al., 2012). No additional filters were applied. Data were down-sampled to $200 \mathrm{~Hz}$ in the BrainVision Analyzer 2.1 and exported to MATLAB. Responses with an onset latency between 1 and $4 \mathrm{~s}$ and a minimum amplitude of $0.02 \mu \mathrm{S}$ were automatically scored using Ledalab (Benedek \& Kaernbach, 2010). If more than one response met the criteria, single responses were summed up. Responses that did not meet the criteria were scored as zero. After Ledalab scoring we used an additional visual inspection of heat maps of single trials and corrected 42 values (out of 240 trials $\times 40$ participants $=9600$ total values) that were over- or underscored by Ledalab. To further correct for interindividual differences and push distribution towards normal we calculated z-values, using the means and standard deviations (SD) over CS and US responses of all learning phases (habituation, acquisition, immediate extinction, and delayed recall) per participant. In accordance with ssVEPs, single-trial data were smoothed with a moving average along the 12 trials (5-point symmetrical, shrinking at the endpoints) within each learning phase and CS-orientation (5 orientations $\times 4$ learning phases with 12 trials each). The z-standardized SCRs were then averaged across the 12 trials of each learning phase and the averages were used in all statistical analyses.

For recording of ECG, we positioned three $\varnothing 8 \mathrm{~mm}$ (inner diameter) electrodes (filled with $5 \% \mathrm{NaCl}$ ECG-paste, GE Medical Systems Information Technologies GmbH, Freiburg, Germany) under the right collarbone, the left shinbone, and (as ground electrode) on the right shinbone.

Systolic and diastolic BP were measured at discrete measurement points using a semiautomatic electronic sphygmomanometer (bosotron 2, Bosch + Sohn, Jungingen, Germany).

427 Therefore, an inflatable cuff was placed around the left upper arm, with the sensor plate positioned over the brachial artery at heart level. 
430

431

432

433

434

435

436

437

438

439

440

441

442

443

444

445

446

447

448

449

450

451

452

453

454

Subjective ratings: Valence, arousal, and US-expectancy

A paper-pencil version of the 9-point pictorial Self-Assessment Manikin (SAM) (Bradley \& Lang, 1994) scale was used to evaluate each CS orientation for its valence (from negative to positive) and arousal (from excited to calm). Ratings were conducted after habituation, acquisition, immediate extinction as well as before and after 24-delayed recall. In addition, we asked the participants to rate their expectancy that an US occurs with the depicted grating with answers ranging from -5 (very certain, no) over 0 (uncertain) to 5 (very certain, yes). Except for after habituation, paper-pencil US-expectancy ratings were conducted together with our SAM ratings.

\section{Overall Procedure}

The study covered two parts: the screening session, lasting about one hour, explaining the general procedure of the main session, testing for inclusion and exclusion criteria and obtaining informed consent (see Participants section for description), and the main conditioning study. Screening and the main study were scheduled on different days.

Main conditioning study:

The main session was conducted on two consecutive days, starting at $10 \mathrm{am}, 14 \mathrm{pm}$ or 17:30 pm. The duration of day 1 and day 2 of the main session were 2 hours and 1 hour, respectively. At the beginning of day 1 and day 2, we attached EEG, ECG, EOG, SCR electrodes and positioned the inflatable cuff for BP measures. Habituation, acquisition, and immediate extinction took place on day 1 , while a 24-h delayed recall took place on day 2 . After each learning phase on day 1 (i.e., after habituation, acquisition, extinction) as well as before and after delayed recall on day 2, resting periods, SAM and US expectancy ratings (except after habituation, where SAMs were conducted without US expectancy ratings, since no US has occurred), and ECG, SCR, as well as BP measures were done (see Fig. 1B). 
Before starting the computer task, we read standardized "general information about the experiment", including a description of the procedure and the stimuli we were about to present. Subjects were instructed to sit comfortably and avoid any movements (except eye blinking) for the entire computer tasks and the explicitly announced measurement periods. During the resting phases, subjects were encouraged to move carefully to avoid detachment of electrodes. At the beginning of habituation, subjects were asked to fixate an upcoming white cross in the center of the screen followed by some black and white 'flickering' gratings. Prior to acquisition, we informed the participants that a loud 'flickering' noise would be presented with only one of the gratings. However, we did not specify which of the five orientations would predict the aversive noise. Prior to immediate extinction (day 1) and delayed recall (day 2) participants were asked to remember the instructions, without informing them that no aversive noise would be presented.

\section{Statistical analysis}

469 We submitted each of the memory outcome measures (i.e., US-expectancy ratings, affective valence and arousal ratings, SCRs, and SSVEPs) to a $5 \times 2$ repeated measures ANOVA, 471 conducted on SPSS software (SPSS 26.0 Inc, Chicago). The mixed ANOVA included the within-subject factor orientation (i.e., the five CS: $25^{\circ}, 35^{\circ}, 45^{\circ}, 55^{\circ}$, and $65^{\circ}$ ) and the between-subject factor group (i.e., in-phase with $0^{\circ}$ phase offset vs. out-of-phase with $90^{\circ}$, $180^{\circ}$, and $270^{\circ}$ phase offset). To test for the expected form of the orientation effect

475 independent of group, we conducted a custom contrast for Generalization, using contrast 476 weights adapted from prior studies (Figure 4 A, Generalization weights: -0.529, 0.247 , $477 \quad 0.564,0.247$, and -0.529$)$.

As this is the first attempt to compare a synchronized vs. non-synchronized condition with a

479 fear generalization design, we hypothesized that group differences may manifest in one of 480 three possible ways: (1) Synchronized CS-US presentation may lead to major increases in 481 CS-responding not limited to the CS+ (this would be evident in a main effect group in the 
ANOVA). (2) Synchronized CS-US presentation may dramatically change the pattern of responding over the five different CS (this could be evident in an orientation $\times$ group interaction in the ANOVA). (3) Finally, synchronized CS-US presentation may alter the width of the generalization curve. This could optimize learning, resulting in a narrower generalization and thus better discrimination between the five CS, without changing overall response levels or dramatically changing the response pattern. ANOVA interactions would not be able to detect this. Therefore, we designed a custom contrast for the group $\mathrm{x}$ orientation interaction, using the LMATRIX command for contrast coefficient matrices in SPSS. We obtained the contrast weights by subtracting a broader generalization profile

(Figure $4 \mathbf{B}$, orange line and font) from a narrower generalization profile (Figure $4 \mathbf{B}$, blue line and font), resulting in a form resembling a 'Mexican Hat' (weights: 0.142, $-0.489,0.694$, 0.489, and 0.142). We expected group differences to manifest during (or directly after) acquisition. However, to explore the longevity of potential group effects, we repeated our $5 \mathrm{x}$ 2 ANOVA and the custom 'Mexican Hat' group $x$ orientation contrast for extinction, and delayed recall on day 2 .

\section{Table 1.}

Summary of statistical analyses. Table shows statistical analyses including $p$ value and effect size for each memory outcome measure, separated by learning phase. For each outcome measure, we calculated repeated-measures ANOVAs with the CS orientation as within-subject factor and the group (in-phase vs. out-of-phase) as between-subject factor. Successful conditioning, i.e., increased response towards the CS+ respective of group) was validated by main effects of orientations (noted in the column effects as ME: $\mathbf{0}$ ). To account for the specific symmetric generalization pattern (CS+ in the middle), additional generalization contrast fits were used (noted as GEN). Main effects of group (ME: g) and group $\mathrm{x}$ orientation interactions ( $\mathbf{0} \mathbf{x} \mathbf{g}$ INT) addressed differences between in-phase and outof-phase conditioning. Better grating discrimination vs. stronger generalization across orientations are described by a Mexican Hat contrast fit for the group $\mathrm{x}$ orientation interactions (MEX). 


\begin{tabular}{|c|c|c|c|c|c|c|}
\hline & $\begin{array}{l}\text { Data } \\
\text { structure }\end{array}$ & $\begin{array}{l}\text { Type of } \\
\text { test }\end{array}$ & Effects & Statistic & $p$ value & effect size \\
\hline \multicolumn{7}{|c|}{ US-expectancy } \\
\hline & Acquisition & & & & & \\
\hline a & normal & ANOVA & ME: o & $F_{(3,109)}=12.491$ & $6.764 \mathrm{E}-7$ & $\eta_{p}^{2}=0.247$ \\
\hline b & normal & ANOVA & GEN & $F_{(1,38)}=28.360$ & .000005 & $\eta_{p}^{2}=0.427$ \\
\hline C & normal & ANOVA & ME: $g$ & $F_{(1,38)}=7.310$ & .010 & $\eta_{p}^{2}=0.161$ \\
\hline d & normal & ANOVA & $\begin{array}{l}\text { oxg } \\
\text { INT }\end{array}$ & $F_{(3,109)}=1.133$ & .338 & $\eta_{p}^{2}=0.029$ \\
\hline e & $\begin{array}{l}\text { normal } \\
\text { Extinction }\end{array}$ & ANOVA & MEX & $F_{(1,38)}=4.796$ & .035 & $\eta_{p}^{2}=0.112$ \\
\hline$f$ & normal & ANOVA & ME: g & $F_{(1,38)}=0.621$ & .436 & $\eta_{p}^{2}=0.016$ \\
\hline g & normal & ANOVA & $\begin{array}{l}\text { oxg } \\
\text { INT }\end{array}$ & $F_{(3,113)}=1.363$ & .258 & $\eta_{p}^{2}=0.035$ \\
\hline h & normal & ANOVA & MEX & $F_{(1,38)}=6.660$ & .014 & $\eta_{p}^{2}=0.149$ \\
\hline & \multicolumn{6}{|c|}{ Delayed Recall (Day 2) } \\
\hline i & normal & ANOVA & ME: $g$ & $F_{(1,36)}=0.688$ & .412 & $\eta_{p}^{2}=0.019$ \\
\hline j & normal & ANOVA & $\begin{array}{l}\text { oxg } \\
\text { INT }\end{array}$ & $F_{(3,100)}=1.172$ & .323 & $\eta_{p}^{2}=0.032$ \\
\hline k & normal & ANOVA & MEX & $F_{(1.36)}=3.090$ & .087 & $\eta_{p}^{2}=0.079$ \\
\hline \multicolumn{7}{|c|}{ Valence \& Arousal } \\
\hline & Acquisition & & & & & \\
\hline I & normal & ANOVA $_{\text {Val }}$ & ME: o & $F_{(3,96)}=7.756$ & .000272 & $\eta_{p}^{2}=0.170$ \\
\hline $\mathrm{m}$ & normal & ANOVA $_{\text {Aro }}$ & ME: o & $F_{(3,100)}=10.928$ & .000008 & $\eta_{p}^{2}=0.223$ \\
\hline$n$ & normal & ANOVA $_{\text {Val }}$ & GEN & $F_{(1,38)}=12.354$ & .001 & $\eta_{p}^{2}=0.245$ \\
\hline o & normal & ANOVA $_{\text {Aro }}$ & GEN & $F_{(1,38)}=19.587$ & .000078 & $\eta_{p}^{2}=0.340$ \\
\hline $\mathrm{p}$ & normal & ANOVA $_{\text {Val }}$ & ME: $g$ & $F_{(1,38)}=1.221$ & .276 & $\eta_{p}^{2}=0.031$ \\
\hline$q$ & normal & ANOVA $_{\text {Val }}$ & $\begin{array}{l}\text { oxg } \\
\text { INT }\end{array}$ & $F_{(3,96)}=1.502$ & .224 & $\eta_{p}^{2}=0.038$ \\
\hline r & normal & ANOVA $_{\text {Aro }}$ & ME: $g$ & $F_{(1,38)}=1.248$ & .271 & $\eta_{p}^{2}=0.032$ \\
\hline s & normal & ANOVA $_{\text {Aro }}$ & $\begin{array}{l}\text { oxg } \\
\text { INT }\end{array}$ & $F_{(3,100)}=1.658$ & .187 & $\eta_{p}^{2}=0.042$ \\
\hline $\mathrm{t}$ & normal & ANOVA $_{\text {Val }}$ & MEX & $F_{(1,38)}=9.228$ & .004 & $\eta_{p}^{2}=0.195$ \\
\hline $\mathrm{u}$ & $\begin{array}{l}\text { normal } \\
\text { Extinction }\end{array}$ & ANOVA $_{\text {Aro }}$ & MEX & $F_{(1,38)}=7.325$ & .010 & $\eta_{p}^{2}=0.162$ \\
\hline $\mathrm{v}$ & normal & ANOVA $_{\text {Val }}$ & ME: $g$ & $F_{(1,38)}=1.810$ & .186 & $\eta_{p}^{2}=0.045$ \\
\hline w & normal & ANOVA $_{\text {Val }}$ & oxg & $F_{(3,117)}=0.647$ & .590 & $\eta_{p}^{2}=0.017$ \\
\hline
\end{tabular}




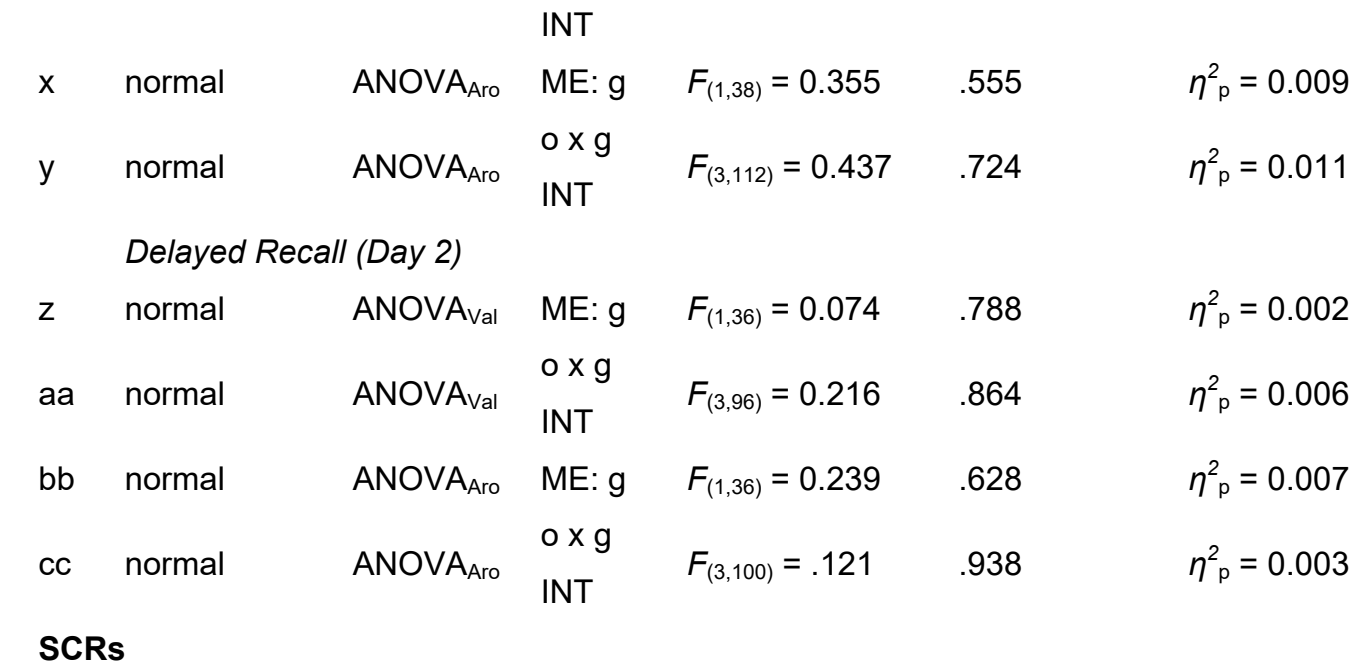

$\begin{array}{lllllll} & \text { Acquisition } & & & & \\ \text { dd } & \text { normal } & \text { ANOVA } & \text { ME: o } & F_{(3,96)}=14.856 & 3.1057 \mathrm{E}-7 & \eta_{p}^{2}=0.281 \\ \text { ee } & \text { normal } & \text { ANOVA } & \text { GEN } & F_{(1,38)}=31.987 & .000002 & \eta_{p}^{2}=0.457 \\ \mathrm{ff} & \text { normal } & \text { ANOVA } & \text { ME: g } & F_{(1,38)}=0.931 & .341 & \eta_{p}^{2}=0.240 \\ \text { gg } & \text { normal } & \text { ANOVA } & \text { oxg } & & & \\ \text { INT } & F_{(3,96)}=0.833 & .461 & \eta_{p}^{2}=0.021\end{array}$

Extinction

hh normal

ANOVA ME: $g$

$F_{(1,38)}=1.170$

.286

$\eta_{p}^{2}=0.030$

ii normal

ANOVA

o $\mathrm{g}$

INT

$F_{(3,117)}=0.921 \quad .435$

$\eta_{p}^{2}=0.024$

Delayed Recall (Day 2)

jj normal ANOVA

ME: $g \quad F_{(1,38)}=0.002 \quad .965$

$\eta_{p}^{2}=0.00005$

kk normal

ANOVA

oxg

INT

$F_{(3,116)}=1.483 \quad .222$

$\eta_{p}^{2}=0.038$

\section{ssVEPs}

Acquisition

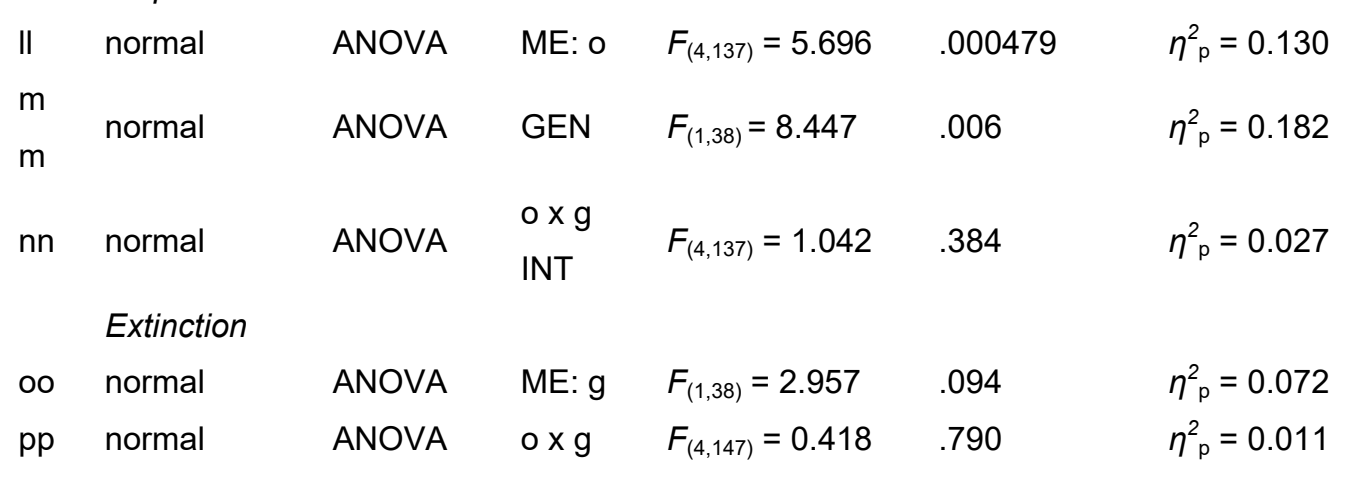


INT

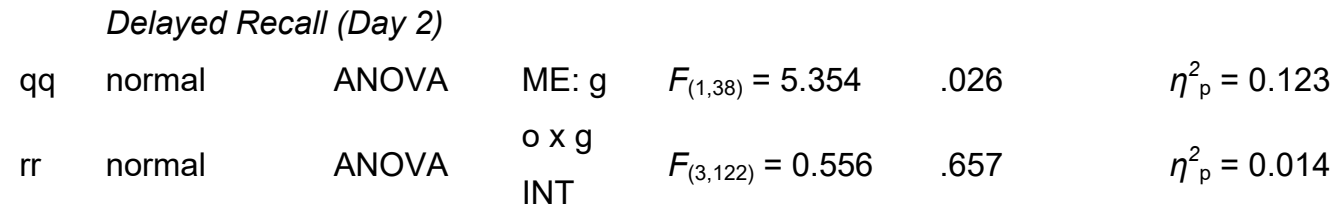

Abbreviations: $A N O V A=$ mixed repeated-measures ANOVA; $M E$ = main effect; $\mathrm{o}=$ orientation; $\eta_{\mathrm{p}}^{2}=$ partial $\eta^{2} ; \mathrm{g}=$ group; $\mathrm{MEX}=$ Mexican Hat contrast fit of orientation $\mathrm{x}$ group interaction; INT = Interaction; GEN = Generalization fit; Val = Valence; Aro = Arousal 499

Results

Phase synchronization causes a better discrimination between CS+ and neighboring CSgratings in the US-expectancy ratings

503

504

505

506

507

508

509

510

511

512

513

514

515

516

517

518

519

We found an effect of orientation on US-expectancies collected immediately after the CS+ was repeatedly aversively reinforced during acquisition $\left(F_{(3,109)}=12.491, p=6.764 \mathrm{E}-7\right.$, part. $\eta^{2}=.247$; Table 1, a). The resulting pattern reflected generalization around the CS+ (Figure 5), with the CS+ and the most similar gratings receiving the highest US-expectancy scores (generalization contrast fit $\left(F_{(1,38)}=28.360, p=.000005\right.$, part. $\eta^{2}=.427$, Table 1, b). In addition, data revealed a main effect of group $\left(F_{(1,38)}=7.310, p=.010\right.$, part. $\eta^{2}=.161$; Table 1, c), but no group $x$ orientation interaction (Table 1, d). Here, the out-of-phase group showed broader generalization of the US-expectancy ratings, while the in-phase group had a narrower generalization pattern with more discrimination between the CS+ and the four CS(Figure 5). This was supported by a significant orientation $\mathrm{x}$ group interaction in the form of a Mexican Hat $\left(F_{(1,38)}=4.796, p=.035\right.$, part. $\eta^{2}=.112$; Table 1, e). As a comprehensive index of CS discrimination (i.e., CS+ versus average of all CS-), we calculated discrimination indices by subtracting the weighted average of CS- responses from the CS+ responses (Extended data Figure 5-1). To account for the fact that the $35^{\circ}$ and $55^{\circ} \mathrm{CS}$ - orientation only differ from the CS+ by $10^{\circ}$ and are thus harder to discriminate, these orientations were multiplied with a weight of $0.33[\ldots]$, before averaging. The more dissimilar orientations $\left(25^{\circ}\right.$, $65^{\circ}$ ) differ by $20^{\circ}$ from the CS+ and are easier to discriminate. Therefore, these two weighted 
with $0.166[\ldots]$, i.e., half of the weight of the more similar orientations. Although the CSweights account for the perceptual difference, they are not directly derived from a

522 psychophysics curve. Figure 5-1 depicts estimation statistics for the discrimination indices within each learning phase by presenting individual values as well as the effect sizes (Hedge's g) as a bootstrap 95\% confidence interval (5000 samples) (Ho et al., 2019). To increase transparency, extended data, Figure 5-2 shows the same for a discrimination index computed with the unweighted average of the four CS-.

527

For US-expectancy ratings collected after extinction, we found no main effect of group or a group $\mathrm{x}$ orientation interaction (Table 1, f, g). However, even after extinction trials the inphase group showed a narrower generalization pattern than the out-of-phase group (Figure 5), Mexican Hat contrast fit for the orientation $x$ group interaction: $\left(F_{(1,38)}=6.660, p=.014\right.$, part. $\eta^{2}=.149$, Table1, h). On day 2, 24 h later (Figure 5 and extended data Figure 5-1, day 2 before delayed recall) we found no group differences in US-expectancy ratings (no main effect group, no orientation $x$ group interaction, Table $\mathbf{1}, \mathbf{i}, \mathbf{j}$ ), and the generalization was no longer significantly narrower in the in-phase group (Mexican Hat orientation $\mathrm{x}$ group interaction, Table 1, k).
--Insert Figure 5 here--
Synchronization leads to a narrower rating pattern towards the CS+ in valence and arousal ratings after fear acquisition
For both, valence and arousal ratings after acquisition (Figure 6), we found a similar prioritization of the CS+ as for US-expectancy (main effect orientation; valence: $F_{(3,96)}=$ 7.756, $p=.000272$, part. $\eta^{2}=.170$, Table 1, I; arousal: $F_{(3,100)}=10.928, p=.000008$, part. $\eta^{2}$ $=.223$, Table 1, m). Again, reflecting generalization around the CS+ (Generalization fit: valence: $F_{(1,38)}=12.352, p=.001$, part. $\eta^{2}=.245$, Table 1, $\mathbf{n}$; arousal: $F_{(1,38)}=19.587, p=$ .000078 , part. $\eta^{2}=.340$, Table 1, o). Here, mixed ANOVA showed no group main effect or orientation $\mathrm{x}$ group interaction for valence (Table 1, p, q) and arousal (Table 1, r, s). 
547 However, in both measures the in-phase group showed a narrower generalization than the 548 out-of-phase group (Figure 6). This was evident in significant orientation $\mathrm{x}$ group interactions 549 in the form of a Mexican Hat for valence $\left(F_{(1,38)}=9.228, p=.004\right.$, part. $\eta^{2}=.195$, Table 1, $\left.\mathbf{t}\right)$ 550 and arousal $\left(F_{(1,38)}=7.325, p=.010\right.$, part. $\eta^{2}=.162$, Table 1, u). The discrimination indices 551 (CS+ versus averaged CS-) as well as estimation plots including individual values and effect 552 sizes are additionally presented in Figure 6-1.

553 After extinction, there were no effects of synchronization in valence (group main effect or 554 orientation by group interaction Table 1, v, w) or arousal (group main effect or orientation by 555 group interaction Table 1, x, y). The same was true for valence and arousal ratings on day 2 556 (group main effect and orientation $\mathrm{x}$ group interaction, valence: Table 1, z, aa; arousal: 557 Table 1, bb, cc).

\section{--Insert Figure 6 here-}

559

SCRs showed the typical increase towards the reinforced CS+ but were unaffected by the synchronization conditions

Figure $7 A, B$ depicts the SCRs on a trial by trial basis, to visualize temporal dynamics of 563 moving-averaged and z-transformed SCRs. In addition, z-values (i.e., without movingaverage) SCRs are presented in Figure 7-1. However, as single trials are subject to noise, SCRs were analyzed using averaged data (Figure $7 \mathrm{C}$ ) as described in the method section. Pairing the CS+ orientation with the aversive US within acquisition, led to the predicted 567 increase of SCR towards the reinforced grating (main effect orientation; $F_{(3,96)}=14.856, p=$ 568 3.1057E-7, part. $\eta^{2}=.281$, Table 1, dd). The response pattern was described by 569 generalization around the CS+ (Generalization fit: $F_{(1,38)}=31.987, p=.000002$, part. $\eta^{2}=$ 570.457 , Table 1, ee). However, this was independent of group (main effect group and 571 orientation $\mathrm{x}$ group interaction, Table 1, ff, gg, see Figure 7-2 for discrimination indices and 572 estimation statistics). Looking at Figure 7 (and Extended Data Figure 7-1), it is unusual that 573 SCRs towards the CS+ seem already increased on the very first trial of acquisition, 
574 independent of the applied smoothing procedure (see Extended Data Figure 7-1 for

575 unsmoothed data). However, explorative analyses of group differences without the first trial

576 did not change the results, i.e., there was still no overall difference between groups and no

577 significant orientation $\mathrm{x}$ group interaction.

578 During extinction, there was no difference between groups (main effect group and orientation

$579 \times$ group interaction, Table 1, hh, ii) and also on day 2 synchronization had no effects (main 580 effect group and orientation $\mathrm{x}$ group interaction, Table 1, jj, kk).

sSVEP-power revealed a tuning towards the visual CS+ that was similar in both groups

584 Figure $8 \boldsymbol{A}, \boldsymbol{B}$ depicts ssVEPs on trial-by-trial basis, to visualize temporal dynamics and the

Extended Data Figure 8-1 shows ssVEP-ratios without moving-average. However, as for the SCRs, ssVEPs were analyzed using averaged data (Figure $8 \mathbf{C}$ ) as described in the method section.

ssVEPs during acquisition revealed a conditioned power increase towards the CS+ and neighboring gratings (main effect orientation, $F_{(4,137)}=5.696, p=.000479$, part. $\eta^{2}=.130$,

Table 1, II). It was described by a generalization pattern around the CS+ (Generalization fit, $F_{(1,38)}=8.447, p=.006$, part. $\eta^{2}=.182$, Table $\left.1, \mathrm{~mm}\right)$. However, this prioritization was not affected by group (orientation $x$ group interaction, Table 1, nn). In similarity to SCRs during acquisition, Figure $8 \mathrm{~A}$ and $\mathrm{B}$ indicate an increased ssVEP ratio towards the CS+ on the very first trial. However, considering the unsmoothed data in Extended Data Figure 8-1, the power increase here seems to be an artifact of the applied smoothing procedure. As depicted in Figure $8 C$, synchronization had also no effects on ssVEPs in extinction. Consequently, we neither found a significant main effect of group nor an orientation $\mathrm{x}$ group interaction (Table 1, oo, pp). Intriguingly, the ssVEP-power during delayed recall on day 2 was generally higher in the in-phase group than in the out-of-phase group $\left(F_{(1,38)}=5.354, p=.026\right.$, part. $\eta^{2}=.123$, 
601 interaction, Table 1, rr) (Figure 8, c). In accordance with ratings and the SCRs, 602 discrimination indices (weighted CS+ minus averaged CS- gratings) and estimation plots, 603 depicting individual values and effects sizes are presented in Extended Data Figure 8-2. --Insert Figure 8 here--

605

606 
608 The formation of associative memories is an elemental aspect of human behavior, but its 609 underlying neurocomputations are largely unknown. One group of theoretical notions has 610 emphasized the role of phase-synchronized oscillations for binding representations of 611 conditioned cues to behavioral outcomes (e.g., Headley \& Paré, 2017). Recent research has 612 increasingly utilized external rhythmic stimulation to test the role of phase relations in specific 613 frequency bands for the formation of working memory (Polanía et al., 2012; Violante et al., 614 2017) and audiovisual associations (Clouter et al., 2017; Wang et al., 2018). Based on these 615 previous findings, we applied this method for the first time in a fear conditioning paradigm. 616 Modulating the phase-shift of a visual CS and aversive auditory US that was presented in the 617 memory-relevant theta frequency allowed us to causally interpret phase synchronization in 618 fear conditioning. To assess the various response systems that are important in fear learning 619 (Lang et al., 2000), we measured skin conductance responses, indexing the physiological 620 arousal of fear, ratings of valence and arousal to capture the subjective evaluation of each 621 stimulus, and US-expectancies which assesses the participant's knowledge of the CS-US 622 association. Additionally, ssVEPs provided information about visuocortical engagement and tuning patterns in sensory processing.

624 The measures we collected in the current study reflect different facets of the associative 625 conditioning process and as such responded differently to the experimental manipulations. In 626 accordance with our hypothesis, synchronized CS-US presentation facilitated the ability to 627 identify the CS+ as the grating that was most likely followed by the aversive US. Remarkably, 628 participants that received synchronized CS-US presentation discriminated the CS+ more 629 precisely from the neighboring CS- gratings (which only differed in an orientation shift of $10^{\circ}$ ). 630 Participants in the out-of-phase group, in contrast, generalized across the CS+ or the most 631 similar CS- gratings. We therefore conclude that the synchronous input of two (multimodal) 632 stimuli stemming from two sensory modalities strengthens the cognitive representation of the 633 CS-US association. 
634 Consistent with the US-expectancies, the subjective valence and arousal ratings reflected 635 the effects of phase synchronization: While participants who received in-phase stimulation 636 were more sensitive to changes in the perceived valence and arousal of the CS+, 637 participants in the out-of-phase group reported generalized arousal and unpleasantness 638 across the CS+ and neighboring CS- gratings. Hence, synchronous input not only sharpens 639 the cognitive representation of CS-US contingency but seems to have a similar influence on 640 the affective evaluation.

641 Surprisingly, there were no corresponding effects in the SCR or ssVEP data. Considering 642 SCR data, during acquisition both groups showed the strongest response towards the CS+ 643 grating, independent of synchronization. Especially in the single-trial data, however, the in644 phase group appears to respond stronger towards the CS+, which seems to be more 645 pronounced in the first trials. We therefore exploratively tested the potential group difference 646 by segmenting the trials into trial blocks ( 3 blocks with 4 trials per block). However, adding 647 this within-factor to our statistical analysis did not reveal any significant differences between 648 the groups in different phases of acquisition. One possible explanation of the higher SCRs in 649 the beginning of acquisition might be the utilized booster sequence (i.e., 5 of the first 7 650 gratings were CS+ gratings). The booster and the applied criterium to not allow more than 651 two consecutive CS of the same orientation might also be the reason for another unusual 652 observation within our SCR results: Irrespective of the factor group and independent of the 653 applied smoothing procedure, SCR towards the CS+ was already increased on the very first 654 trial. While we applied the booster sequence for a better comparability with previous findings 655 (Antov et al., 2020; McTeague et al., 2015), future studies should consider a different 656 approach to minimize similar trial order effect. Nevertheless, there was no differences 657 between the in-phase and out-of-phase group, thus this observation does not change the 658 following interpretations.

659 An effect of phase synchronization was also missing in the ssVEP-data: Although we were 660 able to detect a tuning pattern with the greatest power for the reinforced CS+ grating for $4-\mathrm{Hz}$ 
661

662

663

664

665

666

667

668

669

670

672

673

674

675

676

677

678

679

680

681

682

683

684

685

686

687

688

stimulus presentation as previously described for stimulation in the low beta-range (Antov et al., 2020; McTeague et al., 2015), the pattern did not differ between in-phase and out-ofphase group.

A possible explanation for the observed discrepancies in the different variables could be the involvement of different memory types we might have assessed with our measures. Although fear conditioning is a well-established and widely used paradigm, it is difficult to strictly distinguish the mechanisms behind each response system. For example, skin conductance responses measure physiological arousal during fear learning (although it is not restricted to fear conditioning) and is often considered as measure of the unaware fear reaction, especially dependent on the amygdala (Christopoulos et al., 2019; Knight et al., 2003, 2006; but see also Lovibond \& Shanks, 2002; Sevenster et al., 2014). US-expectancy ratings, on the contrary, are considered to specifically reflect declarative knowledge of the CS-US contingency (Boddez et al., 2013), which is known to include additional brain structures like the hippocampus. Bechara et al. (1995) observed a neural dissociation between implicit and explicit aspects of a fear conditioning procedure. While patients with bilateral amygdala lesions were unable to elicit SCRs but had an intact memory for the declarative facts, patients with bilateral lesions of the hippocampus showed the exact opposite effects, i.e., they acquired SCRs but failed to recall declarative facts. Speculating that the effects of visual-auditory stimulation is not only restricted to early sensory cortices, but influences deeper brain regions in the course of rhythmic processing, our results might be explainable based on these distinct systems: theta phase synchronization might especially modulate the path involved in forming declarative facts about the CS-US pairing (i.e., US-expectancy ratings), probably including the hippocampus, without influencing the emotional conditioning comprising the amygdala. One possible mechanism could be that the phase-synchronous visual CS+ and auditory US simultaneously arrives at neural populations in the hippocampus, increasing the likelihood of long-term potentiation and thereby enhancing synaptic strength (Fell \& Axmacher, 2011). Although the EEG method used here does not allow drawing conclusions about mechanisms at the synaptic level in subcortical structures, one might also 
689

690

691

692

693

694

695

696

697

698

699

700

701

702

703

704

705

706

707

708

709

710

711

712

713

714

715

716

speculate why the heightened CS-US association in the rating data is not reflected in metrics thought to reflect limbic processing, i.e., the SCRs. There are two potential explanations that we wish to highlight in this context: 1) In various species, theta-phase synchronization has predominantly been examined in the context of LTP in the hippocampus (e.g., Buzsáki, 2002; Huerta \& Lisman, 1995; Hyman et al., 2003; Lega et al., 2012), which is specifically relevant in the formation of declarative memory (Clouter et al., 2017; Eichenbaum, 1999; Wang et al., 2018). Thus, one may speculate that theta-phase synchronization is linked to hippocampusdependent processes, whereas the exact timing of CS and US may play a lesser role in amygdala-dependent fear learning. However, some studies have found theta-phase synchronization between the amygdala and other important structures of the fear circuit (e.g., hippocampus, ventrolateral PFC, anterior gyrus cinguli) as well as within the subnuclei of the amygdala (Karalis et al., 2016; Seidenbecher et al., 2003; Taub et al., 2018; Zheng et al., 2019; for reviews see Bocchio et al., 2017; Çalişkan \& Stork, 2018) during different stages of the fear conditioning process, supporting the general influence of theta synchronization during fear memory formation. As such, future work may wish to characterize the role of synchronization within and between specific brain regions for the establishing and maintenance of fear memories. 2) More importantly, however, is the question if synchronized theta rhythms propagate to the amygdala. Sensory information reaches the amygdala via multiple pathways among which some are faster and sub-cortical or "low" route and others are slower or "high" cortical routes (Silverstein \& Ingvar, 2015). Since our method of visual and auditory synchronized (vs. asychronized) theta stimulation is delivered globally and is unlikely to target one specific pathway, the timing might not have been suitable to enable locally specific synchronization. Considering that we used a generalization paradigm with similar CS gratings, we may offer the speculation that the challenging discrimination of the CS+ requires a more demanding processing via the slow, cortical route, while the simple aversive US reaches the amygdala via the fast, subcortical pathway. Thus, the $40 \mathrm{~ms}$ we added to the US might have been insufficient to achieve theta-synchronization when the CS and US reaches the LA. Due to the relatively long CS-US overlap of 2 seconds, we 
717 additionally cannot rule out that our synchronized stimulation reached the amygdala via the

718 thalamic route first, but then also via cortical routes, leading to cancellation of the first CS-US

719 phase synchronization, hence minimizing the suggested effects.

720 Another interesting consideration in this context is the role of theta synchronization between

721 the amygdala and hippocampus for pattern separation of emotional images: Examining pre-

722 surgical epilepsy patients, Zheng et al. (2019) found that bidirectional theta synchronization

723 between both structures was associated with the ability to discriminate an encoded image

724 and a new, but similar 'lure' image in a test phase. Considering that most of our results

725 consists in a better discrimination between the aversive CS+ and the most similar CS-

726 gratings, synchronized CS-US presentation might be beneficial for the amygdalo-

727 hippocampal communication, associated with enhanced discrimination of emotional content.

728 However, further research with additional outcome measures is needed to pinpoint all underlying neurophysiological processes. For example, future studies may attempt to experimentally untangle declarative and non-declarative memory processes involved in fear conditioning, including their reactivity to synchronized presentation. Measuring amygdala and hippocampal activity via fMRI or in experimental animals may also help to clarify the influence of synchronized presentation on distinct sub-processes of fear conditioning and their associated neural substrates.

One important consideration when interpreting the current results is the fact that group differences were mostly restricted to the encoding phase of fear (acquisition), although we expected that improved fear learning after synchronous presentation prompts greater extinction resistance. Contrary to expectations, we did not find extinction-resistant patterns in the in-phase group during immediate extinction or delayed recall. However, using a reinforcement rate of $100 \%$ is known to cause rapid extinction (e.g., Dunsmoor et al., 2007;

741 Haselgrove et al., 2004), which could make it harder to detect between-group effects.

742 Moreover, because extinction leads to the formation of a new (i.e., CS-noUS) memory trace

743 that inhibits the original fear memory, future research may wish to employ a second CS+ 
744 stimulus that is not extinguished, which will aid in assessing the long-lasting effects of theta-

745 phase synchronization on fear memory recall (i.e., the trace that was causally manipulated

746 by theta-synchronized stimulation). Additionally, animal and human work suggests that

747 prolonged stimulus-free periods during encoding are associated with the more effective

748 production of long-term memory (Jiang et al., 2020; Philips et al., 2013). Increasing the inter-

749 trial-intervals (ITI) might help to form more robust memory traces that persist over time.

750 Another possible cause for the absence of long-term effects of stimulation phase is that the externally modulated CS-US stimulation only affects short-term or working memory processes but does not have any effects on actual long-term memory. In two comparable studies focusing on working memory, Clouter et al. (2017) and their follow-up study by Wang et al. (2018) used a distractor task as a time gap between encoding and recall of the learned video-tone associations, which only lasted for 30 seconds, likely too short to inform the formation of long-term memory.

Although the current study provided evidence of a causal role of theta-phase synchronization in the context of fear conditioning, there are some limitations to consider. First, our sample size was chosen to detect medium to strong effect sizes, which was based on previous studies (Clouter et al., 2017; Wang et al., 2018). Therefore, we cannot rule out the possibility that we could not detect small effect sizes. This is especially interesting for the SCR data, where the responses are descriptively stronger after in-phase CS-US presentation, but the statistics did not show significant differences. Increasing the statistical power via a greater sample size might help to even detect small effect sizes.-Second, we cannot conclude that the stimulation effects observed here are specific to the theta band, because we did not test other frequencies. However, both animal model studies that examined theta-phase synchronization in the fear network (e.g., Seidenbecher et al., 2003; Taub et al., 2018), as well as entrainment studies that focused on working and declarative memory (e.g., Alekseichuk et al., 2016; Clouter et al., 2017; Violante et al., 2017) support the current conclusion that synchronization in the theta-frequency band is specifically important for fear memory formation. Third, we did not explicitly ask whether participants were able to detect 
772

773

774

775

776

777

778

779

780

781

782

783

784

785

786

787

788

789

790

791

792

793

794

795

796

797

798

799

the synchronous or asynchronous timing between CS and US, and therefore we cannot rule out that out-of-phase or in-phase stimulation exerted effects based on phenomenological, perceptual differences. Nevertheless, we used the exact time lags utilized by both, Clouter et al. (2017) as well as Wang et al. (2018) who did not observe any interference with perceptual judgments or decline in performance. In addition, Clouter et al. (2017) conducted a control experiment with static stimuli which, on a perceptual basis, represents the best-case scenario for perceptual binding and still found better results after theta-synchronized videoaudio presentation. Fourth, although EEG data showed a group-independent tuning towards the CS+ grating, supporting the conditioned effects on sensory processing, the hypothesized sharpening in the in-phase group was not confirmed. What we found is a general increase in ssVEP-power in the in-phase group during day 2, suggesting a stronger engagement of the sensory cortex. However, based on the present data, we cannot establish to what extent this effect was caused by the theta-phase synchronization on day 1 as opposed to arising as an epiphenomenon, e.g., of the cognitive changes induced by the synchronization. Fifth, thetasynchronization may not facilitate learning, but desynchronized stimulation may disrupt ongoing oscillatory processes, resulting in less precise (i.e., more generalized) fear responses (Alekseichuk et al., 2017). To clarify this assumption, future work may include a third group in which participants are presented with non-flickering CS and US stimuli.

Finally, an important limitation is that we were not able to show that participants' auditory and visual EEG responses were synchronized or de-synchronized as intended. This was due to limited number of trials and noisy US-data. In contrast to previous work with innocuous stimuli, the US in a fear conditioning experiment has to be highly aversive. Inherently, this means that the duration of the US (the only period where auditory and visual stimulation overlap) will produce noisy EEG-data with many movement, startle-response, and other artifacts. This is why EEG studies of human fear conditioning (regardless of the US used and of the of number trials) typically do not analyze any data during US-presentation window. Because the US is aversive, we did not want to expose participants to more noise than absolutely necessary. Thus, based on previous experiments, we limited the duration of a 
800

801

802

803

804

805

806

807

808

809

810

811

812

813 single US to 2000 ms and the US trials to 12 per participant. In combination with inherently noisy EEG-data during a US-presentation precluded us from localizing and analyzing phase differences in the brain response. This should be addressed with a modified design in future studies. Nevertheless, we did verify the precise nature of the bi-modal stimulation on a single-trial and single-subject level (Figure 2). Importantly, earlier work (Clouter et al., 2017; Wang et al., 2018) with 4-Hz audio-video synchronization using larger trial numbers and nonaversive audio stimulation have shown that precise audio-video stimulation results in synchronized responding in the auditory and visual cortex. Future studies may also consider extending the temporal gap between the acquisition phase and the delayed recall, because previous work has indicated that theta-band synchronization between the amygdala and sensory cortices affects the storage of fear information in remote, but not recent fear retrieval (Do-Monte et al., 2015; Sacco \& Sacchetti, 2010).

In conclusion, the current study represents an initial step towards establishing the causal effects of theta-phase synchronization for fear memory formation. Our results replicate the importance of synchronization for acquiring new cognitive representations, measured via USexpectancy ratings, and affective evaluation (subjective valence and arousal ratings). By contrast, the present evidence was mixed at the level of sympathetic (skin conductance) and visuocortical (ssVEPs) engagement. Future studies may wish to further explore the differentiation between different response systems in the context of fear conditioning. Leveraging the potential of rhythmic stimulation and synchronization while taking into account the evolution of fear acquisition across the learning phases will ultimately assist in improving our understanding of the mechanisms behind the acquisition of learned fear responses. 


\section{References}

Alekseichuk, I., Pabel, S. C., Antal, A., \& Paulus, W. (2017). Intrahemispheric theta rhythm desynchronization impairs working memory. Restorative Neurology and Neuroscience, 35(2), 147-158. https://doi.org/10.3233/RNN-160714

Alekseichuk, I., Turi, Z., Amador de Lara, G., Antal, A., \& Paulus, W. (2016). Spatial working memory in humans depends on theta and high gamma synchronization in the prefrontal cortex. Current Biology, 26(12), 1513-1521. https://doi.org/10.1016/j.cub.2016.04.035

Antov, M. I., Plog, E., Bierwirth, P., Keil, A., \& Stockhorst, U. (2020). Visuocortical tuning to a threat-related feature persists after extinction and consolidation of conditioned fear. Scientific Reports, 10(1), 3926. https://doi.org/10.1038/s41598-020-60597-z

Bechara, A., Tranel, D., Damasio, H., Adolphs, R., Rockland, C., \& Damasio, A. (1995). Double dissociation of conditioning and declarative knowledge relative to the amygdala and hippocampus in humans. Science, 269(5227), 1115-1118. https://doi.org/10.1126/science.7652558

Benchenane, K., Peyrache, A., Khamassi, M., Tierney, P. L., Gioanni, Y., Battaglia, F. P., \& Wiener, S. I. (2010). Coherent theta oscillations and reorganization of spike timing in the hippocampal- prefrontal network upon learning. Neuron, 66(6), 921-936. https://doi.org/10.1016/j.neuron.2010.05.013

Benedek, M., \& Kaernbach, C. (2010). A continuous measure of phasic electrodermal activity. Journal of Neuroscience Methods, 190(1), 80-91. https://doi.org/10.1016/j.jneumeth.2010.04.028

Blair, H. T., Schafe, G. E., Bauer, E. P., Blair, H. T., Schafe, G. E., Bauer, E. P., Rodrigues, S. M., \& Ledoux, J. E. (2001). Synaptic plasticity in the lateral amygdala: a cellular hypothesis of fear conditioning. Learning \& Memory, 8, 229-242. https://doi.org/10.1101//m.30901

Bliss, T. V. P., Collingridge, G. L., Morris, R. G. M., \& Reymann, K. G. (2018). Long-term potentiation in the hippocampus : discovery, mechanisms and function. Neuroforum, 24(3), 103-120.

Bocchio, M., Nabavi, S., \& Capogna, M. (2017). Synaptic plasticity, engrams, and network oscillations in amygdala circuits for storage and retrieval of emotional memories. Neuron, 94(4), 731-743. https://doi.org/10.1016/j.neuron.2017.03.022

Boddez, Y., Baeyens, F., Luyten, L., Vansteenwegen, D., Hermans, D., \& Beckers, T. (2013). Rating data are underrated: Validity of US expectancy in human fear conditioning. Journal of Behavior Therapy and Experimental Psychiatry, 44(2), 201-206. https://doi.org/10.1016/j.jbtep.2012.08.003

Boucsein, W., Fowles, D. C., Grimnes, S., Ben-Shakhar, G., Roth, W. T., Dawson, M. E., \& Filion, D. L. (2012). Publication recommendations for electrodermal measurements. Psychophysiology, 49(8), 1017-1034. https://doi.org/10.1111/j.1469-8986.2012.01384.x

Bradley, M. M., \& Lang, P. J. (1994). Measuring emotion: the Self-Assessment Manikin and the Semantic Differential. Journal of Behavior Therapy and Experimental Psychiatry, 25(1), 49-59. http://www.ncbi.nlm.nih.gov/pubmed/7962581

Brainard, D. H. (1997). The Psychophysics Toolbox. Spatial Vision, 10(4), 433-436. https://doi.org/10.1163/156856897X00357

Buzsáki, G. (2002). Theta oscillations in the hippocampus. Neuron, 33, 1-16.

Çalişkan, G., \& Stork, O. (2018). Hippocampal network oscillations as mediators of 
behavioural metaplasticity: Insights from emotional learning. Neurobiology of Learning and Memory, February. https://doi.org/10.1016/j.nlm.2018.02.022

Christopoulos, G. I., Uy, M. A., \& Yap, W. J. (2019). The body and the brain: measuring skin conductance responses to understand the emotional experience. Organizational Research Methods, 22(1), 394-420. https://doi.org/10.1177/1094428116681073

Clouter, A., Shapiro, K. L., \& Hanslmayr, S. (2017). Theta phase synchronization is the glue that binds human associative memory. Current Biology, 27(20), 3143-3148.e6. https://doi.org/10.1016/j.cub.2017.09.001

Do-Monte, F. H., Quinõnes-Laracuente, K., \& Quirk, G. J. (2015). A temporal shift in the circuits mediating retrieval of fear memory. Nature, 519(7544), 460-463. https://doi.org/10.1038/nature14030

Dunsmoor, J. E., Bandettini, P. A., \& Knight, D. C. (2007). Impact of continuous versus intermittent CS-UCS pairing on human brain activation during Pavlovian fear conditioning. Behavioral Neuroscience, 121(4), 635-642. https://doi.org/10.1037/07357044.121.4.635

Eichenbaum, H. (1999). The hippocampus and mechanisms of declarative memory. Behavioural Brain Research, 103(2), 123-133. https://doi.org/10.1016/S01664328(99)00044-3

Fell, J., \& Axmacher, N. (2011). The role of phase synchronization in memory processes. Nature Reviews Neuroscience, 12(2), 105-118. https://doi.org/10.1038/nrn2979

Foa, E. B. (2000). Psychosocial treatment of posttraumatic stress disorder. The Journal of Clinical Psychiatry, 61 Suppl 5, 43-48. http://www.ncbi.nlm.nih.gov/pubmed/10761678

Fries, P. (2015). Communication through coherence. Neuron, 88(1), 220-235. https://doi.org/10.1016/j.neuron.2015.09.034

HansImayr, S., Axmacher, N., \& Inman, C. S. (2019). Modulating human memory via entrainment of brain oscillations. Trends in Neurosciences, 1-15. https://doi.org/10.1016/j.tins.2019.04.004

Haselgrove, M., Aydin, A., \& Pearce, J. M. (2004). A partial reinforcement extinction effect despite equal rates of reinforcement during pavlovian conditioning. Journal of Experimental Psychology: Animal Behavior Processes, 30(3), 240-250. https://doi.org/10.1037/0097-7403.30.3.240

Headley, D. B., \& Paré, D. (2017). Common oscillatory mechanisms across multiple memory systems. Npj Science of Learning, 2(1), 1. https://doi.org/10.1038/s41539-016-0001-2

Herrmann, C. S., Strüber, D., Helfrich, R. F., \& Engel, A. K. (2016). EEG oscillations: From correlation to causality. International Journal of Psychophysiology, 103, 12-21. https://doi.org/10.1016/j.jpsycho.2015.02.003

Herry, C., \& Johansen, J. P. (2014). Encoding of fear learning and memory in distributed neuronal circuits. Nature Neuroscience, 17(12), 1644-1654. https://doi.org/10.1038/nn.3869

Ho, J., Tumkaya, T., Aryal, S., Choi, H., \& Claridge-Chang, A. (2019). Moving beyond P values: data analysis with estimation graphics. Nature Methods, 16(7), 565-566. https://doi.org/10.1038/s41592-019-0470-3

Huerta, P. T., \& Lisman, J. E. (1995). Bidirectional synaptic plasticity induced by a single burst during cholinergic theta oscillation in CA1 in vitro. Neuron, 15(5), 1053-1063. https://doi.org/10.1016/0896-6273(95)90094-2

Hyman, J. M., Wyble, B. P., Goyal, V., Rossi, C. A., \& Hasselmo, M. E. (2003). Stimulation in 
hippocampal region CA1 in behaving rats yields long-term potentiation when delivered to the peak of theta and long-term depression when delivered to the trough. Journal of Neuroscience, 23(37), 11725-11731. https://doi.org/10.1523/JNEUROSCI.23-3711725.2003

Jiang, L., Wang, L., Yin, Y., Huo, M., Liu, C., Zhou, Q., Yu, D., Xu, L., \& Mao, R. (2020). Spaced Training Enhances Contextual Fear Memory via Activating Hippocampal 5HT2A Receptors. Frontiers in Molecular Neuroscience, 12(January), 1-8. https://doi.org/10.3389/fnmol.2019.00317

Junghöfer, M., Elbert, T., Leiderer, P., Berg, P., \& Rockstroh, B. (1997). Mapping EEGPotentials on the surface of the brain: A strategy for uncovering cortical sources. Brain Topography, 9(3), 203-217. https://doi.org/10.1007/bf01190389

Karalis, N., Dejean, C., Chaudun, F., Khoder, S., R Rozeske, R., Wurtz, H., Bagur, S., Benchenane, K., Sirota, A., Courtin, J., \& Herry, C. (2016). 4-Hz oscillations synchronize prefrontal-amygdala circuits during fear behavior. Nature Neuroscience, 19(4), 605-612. https://doi.org/10.1038/nn.4251

Kim, W. Bin, \& Cho, J. (2017). Encoding of discriminative fear memory by input-specific LTP in the amygdala. Neuron, 95(5), 1129-1146. https://doi.org/10.1016/j.neuron.2017.08.004

King, A. J., \& Palmer, A. R. (1985). Integration of visual and auditory information in bimodal neurones in the guinea-pig superior colliculus. Experimental Brain Research, 60(3), 492-500. https://doi.org/10.1007/BF00236934

Kleiner, M., Brainard, D., Pelli, D., Ingling, A., Murray, R., \& Broussard, C. (2007). What's new in Psychtoolbox-3 ? Perception, 36(ECVP Abstract Supplement).

Knight, D. C., Nguyen, H. T., \& Bandettini, P. A. (2003). Expression of conditional fear with and without awareness. Proceedings of the National Academy of Sciences of the United States of America, 100(25), 15280-15283. https://doi.org/10.1073/pnas.2535780100

Knight, D. C., Nguyen, H. T., \& Bandettini, P. A. (2006). The role of awareness in delay and trace fear conditioning in humans. Cognitive, Affective and Behavioral Neuroscience, 6(2), 157-162. https://doi.org/10.3758/CABN.6.2.157

Lang, P. J., Davis, M., \& Ohman, A. (2000). Fear and anxiety: animal models and human cognitive psychophysiology. Journal of Affective Disorders, 61(3), 137-159. https://doi.org/10.1016/S0165-0327(00)00343-8

LeDoux, J. E. (2000). Emotion circuits in the brain. Annual Review of Neuroscience, 23(1), 155-184. https://doi.org/10.1146/annurev.neuro.23.1.155

Lega, B. C., Jacobs, J., \& Kahana, M. (2012). Human hippocampal theta oscillations and the formation of episodic memories. Hippocampus, 22(4), 748-761. https://doi.org/10.1002/hipo.20937

Lennie, P. (1981). The physiological basis of variations in visual latency. Vision Research, 21(6), 815-824. https://doi.org/10.1016/0042-6989(81)90180-2

Lovibond, P. F., \& Shanks, D. R. (2002). The role of awareness in Pavlovian conditioning: Empirical evidence and theoretical implications. Journal of Experimental Psychology: Animal Behavior Processes, 28(1), 3-26. https://doi.org/10.1037/0097-7403.28.1.3

Luo, Q., Holroyd, T., Majestic, C., Cheng, X., Schechter, J., \& James Blair, R. (2010). Emotional automaticity is a matter of timing. Journal of Neuroscience, 30(17), 58255829. https://doi.org/10.1523/JNEUROSCI.BC-5668-09.2010

Lynch, M. A. (2004). Long-term potentiation and memory. Physiological Reviews, 84, 87- 
1000 1001

1002

1003

1004

1005

1006

Maren, S., \& Fanselow, M. (1995). Synaptic plasticity in the basolateral amygdala induced by hippocampal formation stimulation in vivo. The Journal of Neuroscience, 15(11), 75487564. https://doi.org/10.1523/JNEUROSCI.15-11-07548.1995

Markram, H., Lübke, J., Frotscher, M., \& Sakmann, B. (1997). Regulation of synaptic efficacy by coincidence of postsynaptic APs and EPSPs. Science, 275(5297), 213-215. https://doi.org/10.1126/science.275.5297.213

McFadyen, J., Mermillod, M., Mattingley, J. B., Halász, V., \& Garrido, M. I. (2017). A rapid subcortical amygdala route for faces irrespective of spatial frequency and emotion. Journal of Neuroscience, 37(14), 3864-3874. https://doi.org/10.1523/JNEUROSCI.3525-16.2017

McTeague, L. M., Gruss, L. F., \& Keil, A. (2015). Aversive learning shapes neuronal orientation tuning in human visual cortex. Nature Communications, 6, 7823. https://doi.org/10.1038/ncomms8823

Moratti, S., \& Keil, A. (2005). Cortical activation during Pavlovian fear conditioning depends on heart rate response patterns: An MEG study. Cognitive Brain Research, 25(2), 459471. https://doi.org/10.1016/j.cogbrainres.2005.07.006

Nokia, M. S., Waselius, T., Mikkonen, J. E., Wikgren, J., \& Penttonen, M. (2015). Phase matters: responding to and learning about peripheral stimuli depends on hippocampal $\theta$ phase at stimulus onset. Learning \& Memory, 22, 307-317. https://doi.org/10.1101//m.038166.115

Orsini, C. A., \& Maren, S. (2012). Neural and cellular mechanisms of fear and extinction memory formation. Neuroscience \& Biobehavioral Reviews, 36(7), 1773-1802. https://doi.org/10.1016/j.neubiorev.2011.12.014

Philips, G. T., Kopec, A. M., \& Carew, T. J. (2013). Pattern and predictability in memory formation: From molecular mechanisms to clinical relevance. Neurobiology of Learning and Memory, 105, 117-124. https://doi.org/10.1016/j.nIm.2013.05.003

Place, R., Farovik, A., Brockmann, M., \& Eichenbaum, H. (2016). Bidirectional prefrontalhippocampal interactions support context-guided memory. Nature Neuroscience, 19(8), 992-994. https://doi.org/10.1038/nn.4327

Polanía, R., Nitsche, M. A., Korman, C., Batsikadze, G., \& Paulus, W. (2012). The importance of timing in segregated theta phase-coupling for cognitive performance. Current Biology, 22(14), 1314-1318. https://doi.org/10.1016/j.cub.2012.05.021

Quirk, G. J., Repa, J. C., \& LeDoux, J. E. (1995). Fear conditioning enhances short-latency auditory responses of lateral amygdala neurons: Parallel recordings in the freely behaving rat. Neuron, 15(5), 1029-1039. https://doi.org/10.1016/0896-6273(95)90092-6

Romanski, L. M., Clugnet, M. C., Bordi, F., \& LeDoux, J. E. (1993). Somatosensory and auditory convergence in the lateral nucleus of the amygdala. Behavioral Neuroscience, 107(3), 444-450. https://doi.org/10.1037/0735-7044.107.3.444

Sacco, T., \& Sacchetti, B. (2010). Role of secondary sensory cortices in emotional memory storage and retrieval in rats. Science, 329, 649-656. https://doi.org/10.1126/science.1183165

Seidenbecher, T., Laxmi, T. R., Stork, O., \& Pape, H. (2003). Amygdalar and hippocampal theta rhythm synchronization during fear memory retrieval. Science, 301(5634), 846850. https://doi.org/10.1126/science.1085818

Sevenster, D., Beckers, T., \& Kindt, M. (2014). Fear conditioning of SCR but not the startle 
reflex requires conscious discrimination of threat and safety. Frontiers in Behavioral Neuroscience, 8. https://doi.org/10.3389/fnbeh.2014.00032

Silverstein, D. N., \& Ingvar, M. (2015). A multi-pathway hypothesis for human visual fear signaling. Frontiers in Systems Neuroscience, 9(AUGUST), 1-20. https://doi.org/10.3389/fnsys.2015.00101

Skrandies, W. (2007). The effect of stimulation frequency and retinal stimulus location on visual evoked potential topography. Brain Topography, 20(1), 15-20. https://doi.org/10.1007/s10548-007-0026-1

Steil, R., \& Ehlers, A. (2000). Posttraumatische Diagnoseskala (PDS). Psychologisches Institut, Universität Jena.

Summerfield, C., \& Mangels, J. A. (2005). Coherent theta-band EEG activity predicts itemcontext binding during encoding. Neurolmage, 24(3), 692-703. https://doi.org/10.1016/j.neuroimage.2004.09.012

Taub, A. H., Perets, R., Kahana, E., \& Paz, R. (2018). Oscillations synchronize amygdala-toprefrontal primate circuits during aversive learning. Neuron, 97(2), 291-298.e3. https://doi.org/10.1016/j.neuron.2017.11.042

Thut, G., Schyns, P. G., \& Gross, J. (2011). Entrainment of perceptually relevant brain oscillations by non-invasive rhythmic stimulation of the human brain. Frontiers in Psychology, 2(170), 1-10. https://doi.org/10.3389/fpsyg.2011.00170

Violante, I. R., Li, L. M., Carmichael, D. W., Lorenz, R., Leech, R., Hampshire, A., Rothwell, J. C., \& Sharp, D. J. (2017). Externally induced frontoparietal synchronization modulates network dynamics and enhances working memory performance. ELife, 6, 1-22. https://doi.org/10.7554/eLife.22001

Wang, D., Clouter, A., Chen, Q., Shapiro, K. L., \& Hanslmayr, S. (2018). Single-trial phase entrainment of theta oscillations in sensory regions predicts human associative memory performance. The Journal of Neuroscience, 38(28), 6299-6309. https://doi.org/10.1523/JNEUROSCI.0349-18.2018

Weiss, S., \& Rappelsberger, P. (2000). Long-range EEG synchronization during word encoding correlates with successful memory performance. Cognitive Brain Research, 9(3), 299-312. https://doi.org/10.1016/S0926-6410(00)00011-2

Zheng, J., Stevenson, R. F., Mander, B. A., Mnatsakanyan, L., Hsu, F. P. K., Vadera, S., Knight, R. T., Yassa, M. A., \& Lin, J. J. (2019). Multiplexing of theta and alpha rhythms in the amygdala-hippocampal circuit supports pattern separation of emotional information. Neuron, 102(4), 887-898.e5. https://doi.org/10.1016/j.neuron.2019.03.025

\section{Figure, Table, and Extended Data Legends}

\section{Figures}

\section{Figure 1.}

Experimental design: Stimuli, procedure, and the operationalization of in-phase vs. out-of-phase groups. (A) Gabor gratings used as conditioned stimuli (CS). The $45^{\circ}$ grating served as CS+, (paired with the unconditioned stimulus [US] during acquisition). The other 4 served as CS- (never paired with the US). The luminance of each CS was sinusoidally modulated at $4 \mathrm{~Hz}$. The US was a broadband white noise, amplitude modulated at $4 \mathrm{~Hz}$ and presented at a maximum of $96.5 \mathrm{~dB}(\mathrm{~A})$. 
1049

1050

1051

1052

1053

1054

1055

1056

1057

1058

1059

1060

1061

1062

1063

1064

1065

1066

1067

1068

1069

1070

1071

1072

1073

1074

1075

1076

1077

1078

1079

1080

1081

1082

1083

1084

1085

1086

1087

1088

1089

1090

1091

1092

1093

1094

1095

(B) Fear conditioning procedure with the learning phases habituation, fear acquisition and extinction (day1) and delayed recall (day 2). Each CS grating was presented 12 times in each learning phase. The US was only presented during fear acquisition (12 times co-terminating with the CS+). At the end of day 2 the unimodal audio task comprised 75 presentations of the $4 \mathrm{~Hz}$-modulated white noise $(4 \mathrm{~s}$ each) at a non-aversive volume ( $\max =70.4 \mathrm{~dB}[\mathrm{~A}]$ ). Vertical lines below the time line indicate the rating time points. Extended data Figure 1-1 shows the specific trial orders 1 and 2 that were used.

(C) Operationalization of the in-phase group vs. out-of-phase group. Fear conditioning for both groups was identical with the only exception that the in-phase group received the $12 \mathrm{CS}+$ US pairings during acquisition without a phase shift $\left(0^{\circ}\right)$ and the out-of-phase group received the CS+ US pairings with a phase shift of $90^{\circ}, 180^{\circ}$, and $270^{\circ}$ (4 trials each). In (C), the top row shows a simplified depiction of a CS changing luminance at $4 \mathrm{~Hz}$ for $750 \mathrm{~ms}$. The bottom part of (C) shows the first $750 \mathrm{~ms}$ of an overlapping CS+ US presentation for the two groups. The light grey curve shows the luminance of the $\mathrm{CS}+$ (each vertical line shows one step following the monitor's $85 \mathrm{~Hz}$ refresh rate). The black $\left(0^{\circ}\right.$ phase shift), dark grey $\left(90^{\circ}\right)$, yellow $\left(180^{\circ}\right)$, and blue $\left(270^{\circ}\right)$ graphs show a down-sampled representation of the $4 \mathrm{~Hz}$ modulated, white noise US.

\section{Figure 2.}

Processing steps and validation of in-phase vs. out-of-phase stimulation. (A) Processing example (one trial of one participant) of our audio (microphone in front of the participant's speakers) and video signal (photo diode attached to the participants' monitor). Data were segmented relative to the onset of an US (i.e., 12 segments per subject). Before analysis, video-data were shifted $40 \mathrm{~ms}$ forward in time to account for the $40 \mathrm{~ms}$ time shift programmed into the stimulus presentation. Data were rectified, bandpass filtered between 3 and $5 \mathrm{~Hz}$ and subjected to a Hilbert transform. Instantaneous phase information at $4 \mathrm{~Hz}$ was extracted from the imaginary part of the analytic signal. (B) Visualization of inphase (left column) and out-of-phase (right column) CS+US stimulation for all CS+ US trials and all participants $(12 \times 20$ trials per group). Each thin orange line shows the video signal of one participant and one trial. Each thin blue line shows the audio signal (one participant and trial). In (B), the top rows show band-pass filtered data, middle row shows the extracted phase information, at the bottom, polar histograms show the clustering of all phase differences per group.

\section{Figure 3.}

ssVEP and ASSR $4 \mathrm{~Hz}$ signal in the time domain and frequency domain, as well as the scalp distribution of the $4 \mathrm{~Hz}$ signal. The signal-to-noise ratio, averaged over all 40 participants (i.e., irrespective of factor group) is presented for the visual (A) and auditory (B) $4 \mathrm{~Hz}$ stimulation.

\section{Figure 4.}

Contrast weights. (A) Generalization weights to test the fit for a generalized fear response towards the CS+ and neighboring CS- orientations, independent of the factor group. (B) Contrast weights (discrimination) to test the group $\mathrm{x}$ orientation interaction. The weights shown for a narrow (blue) and broad (orange) generalization pattern are just examples that if subtracted (narrow - broad) produce the exact discrimination weights we used for the group $\mathrm{x}$ orientation interaction contrast (numbers in black font, $0.142,-0.498,0.694,-0.498,0.142)$, resembling a 'Mexican hat' (black line).

\section{Figure 5.}

US-expectancy ratings separated for each measurement point: after acquisition, after extinction on day 1 , and before delayed recall on day 2 in the in-phase and the out-of-phase group. US-expectancy was rated per CS on scale ranging from -5 (very certain, no US after this CS) over 0 (uncertain) to 5 
1096

1097

1098

1099

1100

1101

1102

1103

1104

1105

1106

1107

1108

1109

1110

1111

1112

1113

1114

1115

1116

1117

1118

1119

1120

1121

1122

1123

1124

1125

1126

1127

1128

1129

1130

1131

1132

1133

1134

1135

1136

1137

1138

1139

1140

1141

(very certain, an US will follow this CS). Each data point presents the mean US-expectancy rating for each CS orientation (averaged over participants per group and measurement point), error bars show one standard error of the mean (SEM).

See Extended data Figure 5-1 for discrimination indices (CS+ minus the weighted average of all CS-) and estimation statistics for US-expectancy ratings. For transparency Extended data Figure 5-2 shows discrimination indices that result when subtracting the unweighted average of the CS- from the CS+.

\section{Figure 6.}

Valence ratings (A) and arousal ratings (B) separated for each measurement point: after habituation, after acquisition, after extinction (day 1), and before delayed recall (day 2). Valence was rated on a 9point SAM scale from 1 (unpleasant) to 9 (pleasant). For better comparability with arousal ratings, valence ratings were recoded, changing the scale from 1 (pleasant) to 9 (unpleasant). Arousal was also rated on a 9-point SAM scale, here ranging from 1 (calm) to 9 (arousing). Each data point presents valence or arousal ratings, respectively, for each CS orientation (averaged over participants per group and measurement point), error bars show one standard error of the mean (SEM). Note: for better visualization $y$-axis is scaled from 3 to 8 instead of showing the full rating range from 1 to 9 . See Extended data Figure 6-1 for discrimination indices (CS+ minus the weighted average of all CS-) and estimation statistics of valence and arousal data. Extended data Figure 6-2 additionally shows the discrimination indices that use the unweighted average of all CS- for subtraction.

\section{Figure 7.}

Single-trial (A, B) and averaged (C) skin conductance responses. Single-trial SCRs are separated by the synchronization condition into the in-phase, i.e., $0^{\circ}$ phase offset $(\mathbf{A})$ and out-of-phase group, i.e., $90^{\circ}, 180^{\circ}, 270^{\circ}$ phase offset (B). Single-trial data are z-transformed SCRs, averaged over participants per group for each trial and CS-orientation. Before averaging, data was smoothed over the the 12 trials of a learning phase using a moving-average (5-points long, symmetrical, shrinking at the endpoints).

(C) depicts averaged data over 12 trials of habituation, acquisition, extinction, and delayed recall to visualize the response patterns within each learning phase. Here, each data point presents ztransformed SCRs of each CS orientation averaged over participants and trials per group.

Z-transformation was calculated with the means and standard deviations (SD) over CS and US responses of all learning phases (habituation, acquisition, immediate extinction, delayed recall) per participant. Error bars show \pm 1 SEM. See Extended data Figure 7-1 for single-trial SCR data without smooting (i.e., no moving-average). Figure 7-2 shows discrimination indices (CS+ minus weighted and average of all CS-) for SCR and estimation statistics. Figure 7-3 depicts discrimination indices without weighting the averaged CS-.

\section{Figure 8.}

Single-trial (A, B) and averaged (C) power of the $4 \mathrm{~Hz}$ steady-state visually evoked potentials (ssVEPs) for each learning phase (habituation, acquisition, extinction, delayed recall). Single-trial data are separated by the synchronization condition into the in-phase, i.e., $0^{\circ}$ phase offset (A) and the outof-phase group, i.e., $90^{\circ}, 180^{\circ}, 270^{\circ}$ phase offset (B).

The ssVEP power is shown as SNR at $4 \mathrm{~Hz}$, corrected for habituation level responding. Correction was done by dividing individual SNR values by the average SNR from habituation (mean over all 60 trials of each participant, disregarding the different CS orientations). Therefore, values larger 1 
1142

1143

1144

1145

1146

1147

1148

1149

1150

1151

1152

1153

1154

1155

1156

1157

1158

1159

1160

1161

1162

1163

1164

1165

1166

1167

describe an enhancement and lower 1 a decrease of ssVEP-SNR at $4 \mathrm{~Hz}$ relative to habituation. Single trial data were smoothed over trials via a moving-average along the 12 trials of each learning phase (5-point symmetrical, shrinking at the endpoints). Each data point in (A) and (B) represents habituation corrected SNR for each trial and CS-orientation, averaged over participants per group.

(C) depicts data averaged over the 12 trials of habituation, acquisition, extinction, and delayed recall to visualize the response patterns within each phase. Error bars show \pm 1 SEM. Note: habituation data in (C) are nearly 'flat' around 1 due to the habituation correction, as described above and in the method section.

Extended Data Figure 8-1 shows single-trial data without the moving-average. Figure 8-2 depicts discrimination indices with weighted CS- averages (CS+ minus weighted average of all CS-) and Figure 8-3 was added for discrimination indices without weighting the averaged CS- responses.

\section{Tables}

\section{Table 1.}

Summary of statistical analyses. Table shows statistical analyses including $p$ value and effect size for each memory outcome measure, separated by learning phase. For each outcome measure, we calculated repeated-measures ANOVAs with the CS orientation as within-subject factor and the group (in-phase vs. out-of-phase) as between-subject factor. Successful conditioning, i.e., increased response towards the CS+ respective of group) was validated by main effects of orientations (noted in the column effects as ME: o). To account for the specific symmetric generalization pattern (CS+ in the middle), additional generalization contrast fits were used (noted as GEN). Main effects of group (ME: g) and group $x$ orientation interactions ( 0 x g INT) addressed differences between in-phase and outof-phase conditioning. Better grating discrimination vs. stronger generalization across orientations are described by a Mexican Hat contrast fit for the group x orientation interactions (MEX). 
1169

1170

1171

1172

1173

1174

1175

1176

1177

1178

1179

1180

1181

1182

1183

1184

1185

1186

1187

1188

1189

1190

1191

1192

1193

1194

1195

1196

1197

1198

1199

1200

1201

1202

1203

1204

1205

1206

1207

1208

1209

1210

1211

1212

1213

\section{Figure 1-1.}

Table of trial list 1 and 2 for CS presentation order within each learning phase. The table shows the sequential order of conditioned stimulus (CS) presentation across the 60 trials of each learning phase. CS were Gabor-Gratings differing only in orientation (orientation degrees are shown in the $2^{\text {nd }}$ to last columns). The $1^{\text {st }}$ column (Trial) shows the sequential number (e.g., trial 2 was the $2^{\text {nd }}$ CS seen by a participant in the specified learning phase). Each participant within the in-phase and out-of-phase groups was randomly assigned to receive stimuli according to list 1 or 2 . Assignment to list 1 and 2 was balanced across groups.

\section{Figure 5-1.}

Weighted discrimination indices for US-expectancy ratings. US-expectancy ratings were first ztransformed within each participant using the mean and SD of all US-expectancy ratings of a participant. With the z-transformed data we computed a weighted discrimination index per learning phase as the difference between the rating of the reinforced $45^{\circ}(\mathrm{CS}+)$ grating and the weighted average of the four CS- gratings. Weights for the CS- correspond to the angular difference in orientation between the four CS- $\left(25^{\circ}, 35^{\circ}, 55^{\circ}, 65^{\circ}\right)$ and the CS $+\left(45^{\circ}\right)$. The two more similar CS- $( \pm$ $10^{\circ}$ to the $\mathrm{CS}+$ ) were weighted with $0.33[\ldots]$, while the more dissimilar orientations $\left( \pm 20^{\circ}\right.$ to the CS + ) were weighted with $0.166[\ldots]$.

Data and effect sizes are shown as a Cumming estimation plot (http://www.estimationstats.com) Top row: swarm plots show the raw discrimination indices per learning phase (each dot is the discrimination index of one participant). Group statistics are indicated to the right of each swarm as gapped lines (gap = mean, line length = $1 \mathrm{SD}$ ). Bottom row: effect size estimates (Hedges' g, black dots) for the 3 relevant comparisons (in-phase vs. out-of-phase for each learning phase) and their $95 \%$ confidence interval $(\mathrm{Cl}$, vertical error bars).

The unpaired Hedge's $\mathrm{g}$ for acquisition is -0.364 [95.0\% Cl $-0.9810 .315] p=.2578$; for extinction, $0.463[\mathrm{Cl}-1.0890 .205] p=.1532$, and for delayed recall, $-0.249[\mathrm{Cl}-0.9070 .370] p=.4206$.

5000 bootstrap samples were taken for $\mathrm{Cl}$ estimation; the $\mathrm{Cl}$ is bias-corrected and accelerated. The two-sided $P$-values are the likelihoods of observing the effect sizes, if the null hypothesis of zero difference is true. For each permutation $P$-value, 5000 reshuffles of the group labels were performed.

\section{Figure 5-2.}

Unweighted discrimination indices for US-expectancy ratings. US-expectancy ratings were ztransformed within each participant using the mean and SD of all US-expectancy ratings of a participant. The unweighted discrimination index shown is the difference between ratings of the CS+ and the unweighted average of the four CS-. Data and effect sizes are shown as a Cumming estimation plot (http://www.estimationstats.com). See the legend of Extended data Figure 5-1 for a detailed description of a Cumming estimation plot.

The unpaired Hedge's $\mathrm{g}$ for acquisition is -0.306 [95\% $\mathrm{Cl}-0.9280 .375], p=.3356$; for extinction, 0.372 [Cl-1.021 0.289], $p=.2346$, and for delayed recall, -0.198 [Cl -0.842 0.433], $p=.5166 .5000$ bootstrap samples were taken for $\mathrm{Cl}$ estimation; the $\mathrm{Cl}$ is bias-corrected and accelerated. The twosided $P$-values are the likelihoods of observing the effect sizes, if the null hypothesis of zero difference is true. For each permutation $P$-value, 5000 reshuffles of the group labels were performed. 
1214

1215

1216

1217

1218

1219

1220

1221

1222

1223

1224

1225

1226

1227

1228

1229

1230

1231

1232

1233

1234

1235

1236

1237

1238

1239

1240

1241

1242

1243

1244

1245

1246

1247

1248

1249

1250

1251

1252

1253

1254

1255

1256

1257

1258

1259

1260

1261

1262

1263

\section{Figure 6-1.}

Weighted discrimination indices for valence ratings (A) and arousal ratings (B). Valence and arousal ratings were first $z$-transformed within each participant using the mean and SD of all ratings of valence and arousal of a participant, respectively. With the z-transformed data we computed a weighted discrimination index per learning phase as the difference between the reinforced $45^{\circ}$ (CS+) grating and the weighted average of the four CS- gratings. Weights for the CS- correspond to the angular difference in orientation between the four CS- $\left(25^{\circ}, 35^{\circ}, 55^{\circ}, 65^{\circ}\right)$ and the CS+ $\left(45^{\circ}\right)$ : the two more similar CS- $\left( \pm 10^{\circ}\right.$ to the CS +$)$ were weighted with $0.33[\ldots]$, while the more dissimilar orientations $( \pm$ $20^{\circ}$ to the CS+) were weighted with 0.166

Data and effect sizes are shown as a Cumming estimation plot (http://www.estimationstats.com). See the legend of Extended data Figure 5-1 for a detailed description of a Cumming estimation plot.

For valence data (A), the unpaired Hedge's $\mathrm{g}$ for habituation is -0.039 [95.0\% $\mathrm{Cl}-0.6800 .568$ ], $p$ $=.896$; for acquisition, -0.660 [Cl $-1.219-0.048$ ], $p=.0372$, for extinction, -0.291 [Cl -0.9250 .354 ], $p=$ .3522 , and for delayed recall, -0.218 [Cl -0.832 0.423], $p=.4848$. For arousal data (B), the unpaired Hedge's $\mathrm{g}$ for habituation is $-0.296[\mathrm{Cl}-0.9140 .386], p=.3372$; for acquisition, $-0.877[\mathrm{Cl}-1.459$ 0.302 ], $p=.0074$, for extinction, -0.382 [Cl -1.0200 .273 ],$p=.2216$, and for delayed recall, -0.142 [Cl $0.7780 .510], p=.6472 .5000$ bootstrap samples were taken for $\mathrm{Cl}$ estimation; the $\mathrm{Cl}$ is bias-corrected and accelerated. The two-sided $P$-values are the likelihoods of observing the effect sizes, if the null hypothesis of zero difference is true. For each permutation $P$-value, 5000 reshuffles of the group labels were performed.

\section{Figure 6-2.}

Unweighted discrimination indices for valence (A) and arousal (B) ratings. Ratings were $z$-transformed within each participant using the mean and SD of all valence and arousal ratings of a participant, respectively. The unweighted discrimination index shown is the difference between ratings of the CS+ and the unweighted average of the four CS-. Data and effect sizes are shown as a Cumming estimation plot (http://www.estimationstats.com). See the legend of Extended data Figure 5-1 for a detailed description of a Cumming estimation plot.

For valence data (A), the unpaired Hedge's $\mathrm{g}$ for habituation is $0.011[95 \% \mathrm{Cl}-0.6220 .618] p=.9678$; for acquisition, -0.578 [Cl-1.153 0.047], $p=.07$, for extinction, -0.220 [Cl -0.8640 .423 ],$p=.488$, and for delayed recall, -0.218 [Cl $-0.8260 .422], p=.485$. For arousal data (B), the unpaired Hedge's g for habituation is -0.255 [-0.866 0.439], $p=.407$; for acquisition, -0.820 [Cl $-1.424-0.225], p=.0128$, for extinction, -0.361 [Cl -1.0010 .295 ], $p=.2466$, and for delayed recall, -0.141 [Cl -0.7740 .503 ], $p=$ .6512. 5000 bootstrap samples were taken for $\mathrm{Cl}$ estimation; the $\mathrm{Cl}$ is bias-corrected and accelerated. The two-sided $P$-values are the likelihoods of observing the effect sizes, if the null hypothesis of zero difference is true. For each permutation $P$-value, 5000 reshuffles of the group labels were performed.

\section{Figure 7-1.}

Single-trial data of skin conductance responses (SCRs) without smoothing over trials. Same data as in Figure 7 (A, B), plotted without the moving-average over trials. SCRs are separated by learning phase (habituation, acquisition, extinction on day 1 , and delayed recall on day 2) and by the synchronization condition into the in-phase, i.e., $0^{\circ}$ phase offset (A) and out-of-phase group, i.e., $90^{\circ}, 180^{\circ}, 270^{\circ}$ phase offset (B). Error bars show \pm 1 SEM.

\section{Figure 7-2.}

Weighted discrimination indices for averaged for averaged skin conductance responses (SCRs). SCRs were first z-transformed within each participant using the means and SD over CS and US 
responses of all learning phases (habituation, acquisition, extinction, delayed recall). With the ztransformed data we computed a weighted discrimination index per learning phase as the difference between the reinforced $45^{\circ}(\mathrm{CS}+)$ grating and the weighted average of the four CS- gratings. Weights for the CS- correspond to the angular difference in orientation between the four CS- $\left(25^{\circ}, 35^{\circ}, 55^{\circ}\right.$, $\left.65^{\circ}\right)$ and the CS $\left(45^{\circ}\right)$ : the two more similar CS- $\left( \pm 10^{\circ}\right.$ to the CS + ) were weighted with $0.33[\ldots]$, while the more dissimilar orientations $\left( \pm 20^{\circ}\right.$ to the $\left.\mathrm{CS}+\right)$ were weighted with $0.166[\ldots]$.

Data and effect sizes are shown as a Cumming estimation plot (http://www.estimationstats.com). See Extended data Figure 5-1 legend for a detailed plot description.

The unpaired Hedge's $\mathrm{g}$ for habituation is -0.249 [95.0\% $\mathrm{Cl}-0.8270 .371], p=.451$; for acquisition, 0.405 [Cl -0.9380 .211 ], $p=.2044$, for extinction, 0.847 [Cl 0.277 1.361], $p=.0096$, and for delayed recall, $0.535[\mathrm{Cl}-0.0911 .056], p=.0916$.

\section{Figure 7-3.}

Unweighted discrimination indices for averaged skin conductance responses (SCRs). SCRs were ztransformed within each participant using the means and SD over CS and US responses of all learning phases (habituation, acquisition, extinction, delayed recall). The unweighted discrimination index shown is the difference between SCR to the CS+ and the unweighted average of the four CS.Data and effect sizes are shown as a Cumming estimation plot (http://www.estimationstats.com). See Extended data Figure 5-1 legend for a detailed plot description.

The unpaired Hedge's $\mathrm{g}$ for habituation is -0.146 [95\% Cl $-0.7540 .461], p=.6618$; for acquisition, 0.385 [Cl -0.9200 .230 ], $p=.2296$, for extinction, 0.754 [Cl 0.1971 .259 ], $p=.0212$, and for delayed recall, 0.549 [Cl-0.071 1.059], $p=.0848 .5000$ bootstrap samples were taken for $\mathrm{Cl}$ estimation; the $\mathrm{Cl}$ is bias-corrected and accelerated. The two-sided $P$-values are the likelihoods of observing the effect sizes, if the null hypothesis of zero difference is true. For each permutation $P$-value, 5000 reshuffles of the group labels were performed.

\section{Figure 8-1.}

Single-trial power of the $4 \mathrm{~Hz}$ steady-state visually evoked potentials (SSVEP) without smoothing over trials. Same data as Figure $\mathbf{8}(\boldsymbol{A}, \boldsymbol{B})$, plotted without the moving-average over trials. Single-trials are separated by learning phase (habituation, acquisition, extinction on day 1 , and delayed recall on day 2 ) and by the synchronization condition into the in-phase, i.e., $0^{\circ}$ phase offset (A) and out-of-phase group, i.e., $90^{\circ}, 180^{\circ}, 270^{\circ}$ phase offset (B). Error bars show \pm 1 SEM.

\section{Figure 8-2.}

Weighted discrimination indices for steady-state visually evoked potentials (ssVEPs). Within each learning phase, using the habituation corrected SNR at $4 \mathrm{~Hz}$ (Figure $8 \mathrm{C}$ ) we computed a weighted discrimination index per learning phase as the difference between the reinforced $45^{\circ}(\mathrm{CS}+)$ grating and the weighted average of the four CS- gratings. Weights for the CS- correspond to the angular difference in orientation between the four CS- $\left(25^{\circ}, 35^{\circ}, 55^{\circ}, 65^{\circ}\right)$ and the CS+ $\left(45^{\circ}\right)$ : the two more similar CS- $\left( \pm 10^{\circ}\right.$ to the CS +$)$ were weighted with $0.33[\ldots]$, while the more dissimilar orientations $( \pm$ $20^{\circ}$ to the $\mathrm{CS}+$ ) were weighted with $0.166[\ldots]$.

Data and effect sizes are shown as a Cumming estimation plot (http://www.estimationstats.com). See Extended data Figure 5-1 legend for a detailed plot description.

The unpaired Hedge's $\mathrm{g}$ for habituation is 0.008 [95.0\% $\mathrm{Cl}-0.652$ 0.633], $p=.979$; for acquisition, 0.114 [Cl -0.7310 .511 ],$p=.7084$, for extinction, 0.130 [Cl -0.5190 .741 ], $p=.683$, and for delayed recall, 0.054 [Cl $-0.5640 .702], p=.08622 .5000$ bootstrap samples were taken for $\mathrm{Cl}$ estimation; the $\mathrm{Cl}$ is bias-corrected and accelerated. The two-sided $P$-values are the likelihoods of observing the 
effect sizes, if the null hypothesis of zero difference is true. For each permutation $P$-value, 5000 reshuffles of the group labels were performed.

Figure 8-3.

1319 Unweighted discrimination indices for SsVEPs. Here, the discrimination index was computed as the difference between the reinforced $45^{\circ}(\mathrm{CS}+)$ grating and the unweighted average of the four CS-. Data and effect sizes are shown as a Cumming estimation plot (http://www.estimationstats.com). See Extended data Figure 5-1 legend for a detailed plot description. The unpaired Hedge's $\mathrm{g}$ for habituation is -0.074 [95\% Cl -0.708 0.569], $p=.8106$; for acquisition, 0.161 [Cl -0.7740 .464 ], $p=.6074$, for extinction, 0.080 [Cl -0.5610 .706 ], $p=.7948$, and for delayed recall, 0.044 [Cl -0.5790 .687 ],$p=.891 .5000$ bootstrap samples were taken for $\mathrm{Cl}$ estimation; the $\mathrm{Cl}$ is bias-corrected and accelerated. The two-sided $P$-values are the likelihoods of observing the effect sizes, if the null hypothesis of zero difference is true. For each permutation $P$-value, 5000 reshuffles of the group labels were performed. 
Table 1.

Summary of statistical analyses. Table shows statistical analyses including $p$ value and effect size for each memory outcome measure, separated by learning phase. For each outcome measure, we calculated repeated-measures ANOVAs with the CS orientation as within-subject factor and the group (in-phase vs. out-of-phase) as between-subject factor. Successful conditioning, i.e., increased response towards the CS+ respective of group) was validated by main effects of orientations (noted in the column effects as ME: o). To account for the specific symmetric generalization pattern (CS+ in the middle), additional generalization contrast fits were used (noted as GEN). Main effects of group (ME: g) and group $x$ orientation interactions ( $\mathbf{0} \mathbf{x} \mathbf{g}$ INT) addressed differences between in-phase and outof-phase conditioning. Better grating discrimination vs. stronger generalization across orientations are described by a Mexican Hat contrast fit for the group $x$ orientation interactions (MEX).

\begin{tabular}{|c|c|c|c|c|c|c|}
\hline & Data structure & Type of test & Effects & Statistic & $p$ value & effect size \\
\hline \multicolumn{7}{|c|}{ US-expectancy } \\
\hline & Acquisition & & & & & \\
\hline a & normal & ANOVA & ME: o & $F_{(3,109)}=12.491$ & $6.764 \mathrm{E}-7$ & $\eta_{p}^{2}=0.247$ \\
\hline b & normal & ANOVA & GEN & $F_{(1,38)}=28.360$ & .000005 & $\eta_{p}^{2}=0.427$ \\
\hline c & normal & ANOVA & ME: $g$ & $F_{(1,38)}=7.310$ & .010 & $\eta_{p}^{2}=0.161$ \\
\hline d & normal & ANOVA & oxg INT & $F_{(3,109)}=1.133$ & .338 & $\eta_{p}^{2}=0.029$ \\
\hline \multirow[t]{2}{*}{$\mathrm{e}$} & normal & ANOVA & MEX & $F_{(1,38)}=4.796$ & .035 & $\eta_{p}^{2}=0.112$ \\
\hline & Extinction & & & & & \\
\hline f & normal & ANOVA & ME: $g$ & $F_{(1,38)}=0.621$ & .436 & $\eta_{p}^{2}=0.016$ \\
\hline g & normal & ANOVA & oxg INT & $F_{(3,113)}=1.363$ & .258 & $\eta_{p}^{2}=0.035$ \\
\hline \multirow[t]{2}{*}{$\mathrm{h}$} & normal & ANOVA & MEX & $F_{(1,38)}=6.660$ & .014 & $\eta_{p}^{2}=0.149$ \\
\hline & Delayed Recall & ay 2) & & & & \\
\hline i & normal & ANOVA & ME: $g$ & $F_{(1,36)}=0.688$ & .412 & $\eta_{p}^{2}=0.019$ \\
\hline j & normal & ANOVA & oxg INT & $F_{(3,100)}=1.172$ & .323 & $\eta_{p}^{2}=0.032$ \\
\hline k & normal & ANOVA & MEX & $F_{(1.36)}=3.090$ & .087 & $\eta_{p}^{2}=0.079$ \\
\hline \multicolumn{7}{|c|}{ Valence \& Arousal } \\
\hline & Acquisition & & & & & \\
\hline I & normal & ANOVAVal $_{\text {Val }}$ & ME: o & $F_{(3,96)}=7.756$ & .000272 & $\eta_{p}^{2}=0.170$ \\
\hline $\mathrm{m}$ & normal & ANOVA $_{\text {Aro }}$ & ME: o & $F_{(3,100)}=10.928$ & .000008 & $\eta_{p}^{2}=0.223$ \\
\hline$n$ & normal & ANOVA $_{\text {Val }}$ & GEN & $F_{(1,38)}=12.354$ & .001 & $\eta_{p}^{2}=0.245$ \\
\hline o & normal & ANOVA $_{\text {Aro }}$ & GEN & $F_{(1,38)}=19.587$ & .000078 & $\eta_{p}^{2}=0.340$ \\
\hline $\mathrm{p}$ & normal & ANOVA $_{\text {Val }}$ & ME: $g$ & $F_{(1,38)}=1.221$ & .276 & $\eta_{p}^{2}=0.031$ \\
\hline$q$ & normal & ANOVA $_{\text {val }}$ & oxg INT & $F_{(3,96)}=1.502$ & .224 & $\eta_{p}^{2}=0.038$ \\
\hline r & normal & ANOVA $_{\text {Aro }}$ & ME: $g$ & $F_{(1,38)}=1.248$ & .271 & $\eta_{p}^{2}=0.032$ \\
\hline s & normal & ANOVA $_{\text {Aro }}$ & oxg INT & $F_{(3,100)}=1.658$ & .187 & $\eta_{p}^{2}=0.042$ \\
\hline $\mathrm{t}$ & normal & ANOVA $_{\text {Val }}$ & MEX & $F_{(1,38)}=9.228$ & .004 & $\eta_{p}^{2}=0.195$ \\
\hline \multirow[t]{2}{*}{$\mathrm{u}$} & normal & ANOVA $_{\text {Aro }}$ & MEX & $F_{(1,38)}=7.325$ & .010 & $\eta_{p}^{2}=0.162$ \\
\hline & Extinction & & & & & \\
\hline v & normal & ANOVA $_{\mathrm{Val}}$ & ME: g & $F_{(1,38)}=1.810$ & .186 & $\eta_{p}^{2}=0.045$ \\
\hline w & normal & ANOVAval $_{\text {Val }}$ & oxg INT & $F_{(3,117)}=0.647$ & .590 & $\eta_{p}^{2}=0.017$ \\
\hline
\end{tabular}




\begin{tabular}{|c|c|c|c|c|c|c|}
\hline$x$ & normal & ANOVA $_{\text {Aro }}$ & ME: $g$ & $F_{(1,38)}=0.355$ & .555 & $\eta_{p}^{2}=0.009$ \\
\hline \multirow[t]{2}{*}{$y$} & normal & ANOVA $_{\text {Aro }}$ & o x g INT & $F_{(3,112)}=0.437$ & .724 & $\eta_{p}^{2}=0.011$ \\
\hline & \multicolumn{5}{|c|}{ Delayed Recall (Day 2) } & \\
\hline z & normal & ANOVAval $_{\text {Val }}$ & ME: $g$ & $F_{(1,36)}=0.074$ & .788 & $\eta_{p}^{2}=0.002$ \\
\hline aa & normal & ANOVAval $_{\text {V }}$ & oxg INT & $F_{(3,96)}=0.216$ & .864 & $\eta_{p}^{2}=0.006$ \\
\hline $\mathrm{bb}$ & normal & ANOVA $_{\text {Aro }}$ & ME: $g$ & $F_{(1,36)}=0.239$ & .628 & $\eta_{p}^{2}=0.007$ \\
\hline cc & normal & ANOVA $_{\text {Aro }}$ & o x g INT & $F_{(3,100)}=.121$ & .938 & $\eta_{p}^{2}=0.003$ \\
\hline \multicolumn{7}{|c|}{ SCRs } \\
\hline & \multicolumn{6}{|c|}{ Acquisition } \\
\hline dd & normal & ANOVA & ME: o & $F_{(3,96)}=14.856$ & 3.1057E-7 & $\eta_{p}^{2}=0.281$ \\
\hline ee & normal & ANOVA & GEN & $F_{(1,38)}=31.987$ & .000002 & $\eta_{p}^{2}=0.457$ \\
\hline $\mathrm{ff}$ & normal & ANOVA & ME: g & $F_{(1,38)}=0.931$ & .341 & $\eta_{p}^{2}=0.240$ \\
\hline \multirow[t]{2}{*}{ gg } & normal & ANOVA & o x g INT & $F_{(3,96)}=0.833$ & .461 & $\eta_{p}^{2}=0.021$ \\
\hline & \multicolumn{6}{|c|}{ Extinction } \\
\hline hh & normal & ANOVA & ME: $g$ & $F_{(1,38)}=1.170$ & .286 & $\eta_{p}^{2}=0.030$ \\
\hline \multirow[t]{2}{*}{ ii } & normal & ANOVA & o x g INT & $F_{(3,117)}=0.921$ & .435 & $\eta_{p}^{2}=0.024$ \\
\hline & \multicolumn{6}{|c|}{ Delayed Recall (Day 2) } \\
\hline jj & normal & ANOVA & ME: $g$ & $F_{(1,38)}=0.002$ & .965 & $\eta_{p}^{2}=0.00005$ \\
\hline kk & normal & ANOVA & o x g INT & $F_{(3,116)}=1.483$ & .222 & $\eta_{p}^{2}=0.038$ \\
\hline
\end{tabular}

sSVEPs

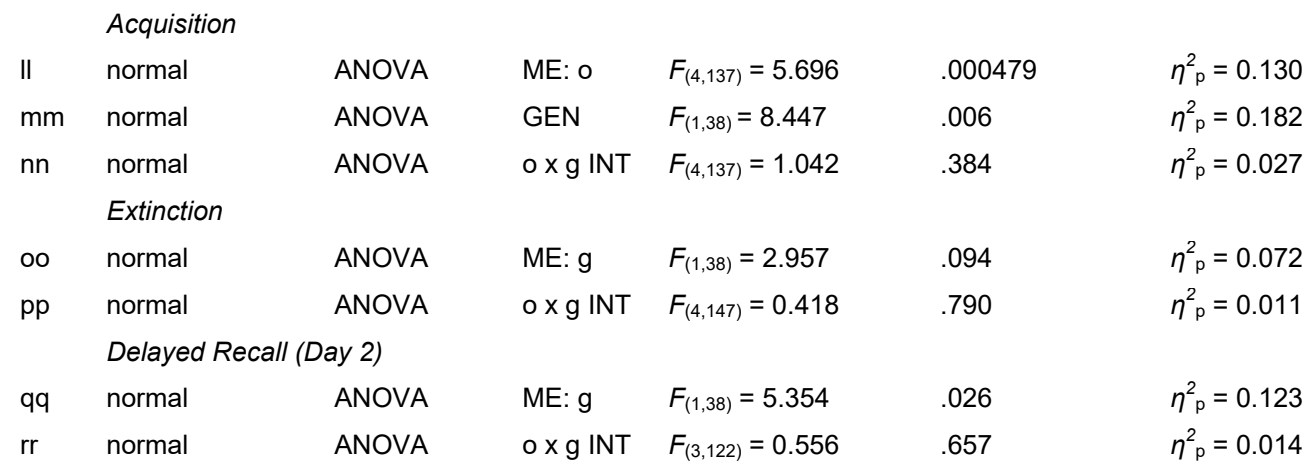

Abbreviations: $A N O V A=$ mixed repeated-measures ANOVA; ME = main effect; $0=$ orientation; $\eta_{p}^{2}=$ partial $\eta^{2} ; g=$ group; MEX = Mexican Hat contrast fit of orientation $x$ group interaction; INT = Interaction; GEN = Generalization fit; Val = Valence; Aro $=$ Arousal 


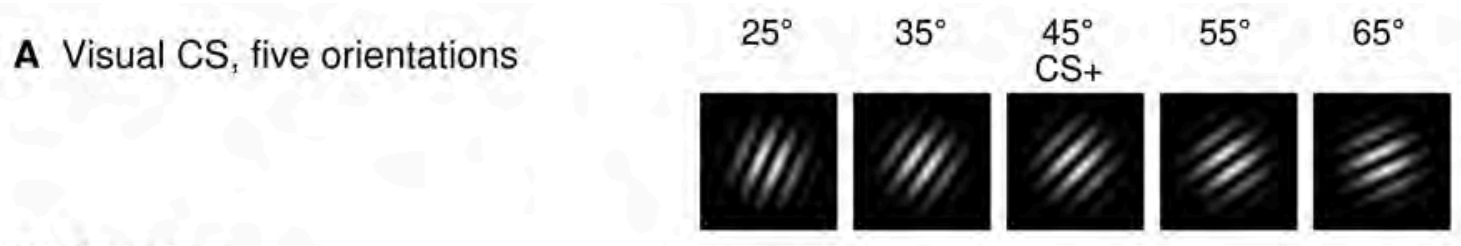

B Procedure

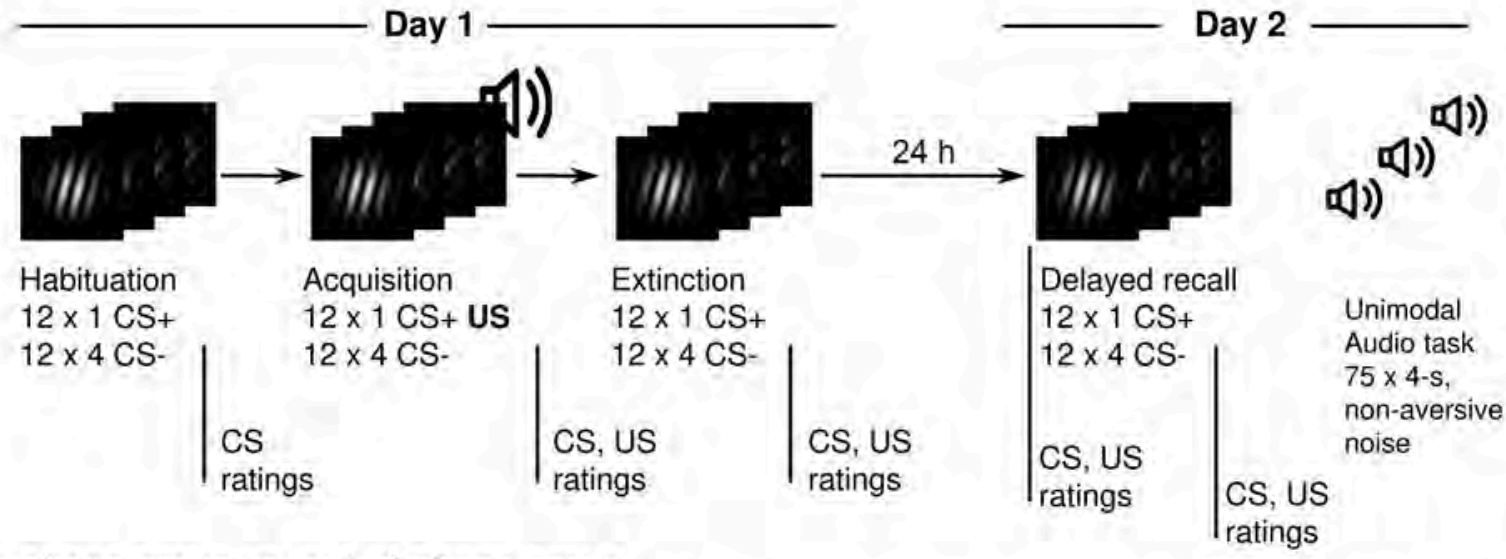

C In-phase group vs. out-of-phase group

Only for the 12 CS+US trials

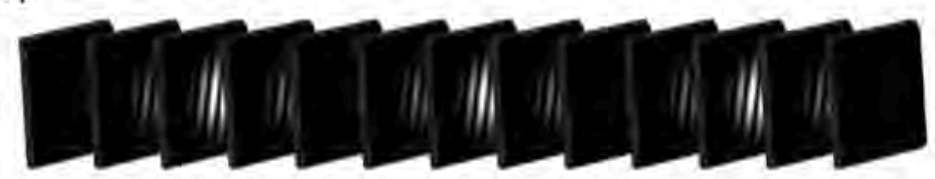

In-phase group $(N=20)$

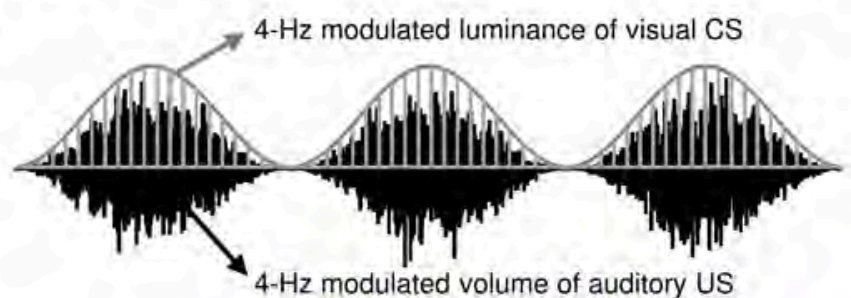

Out-of-phase group $(N=20)$ $90^{\circ}, 180^{\circ}$, and $270^{\circ}$ phase offset

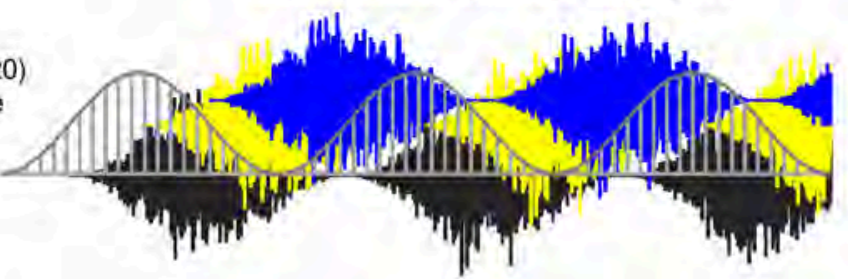




\section{A Processing example}

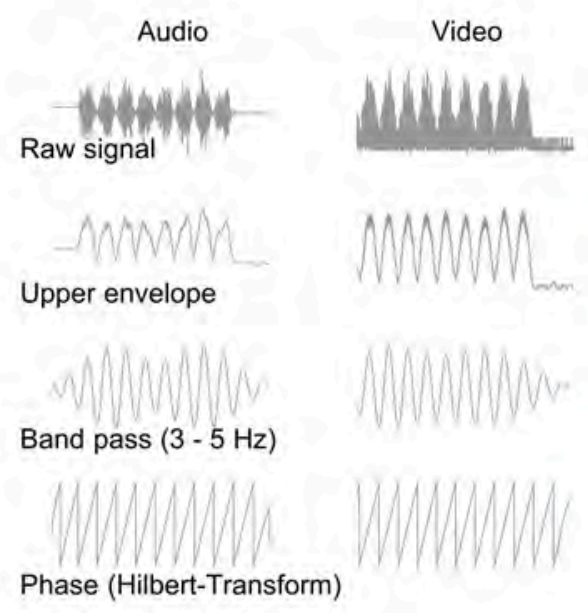

B Stimulation validation

In-phase group

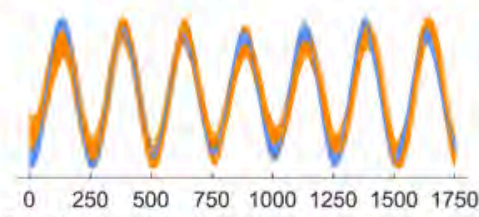
Band pass $(3-5 \mathrm{~Hz})$

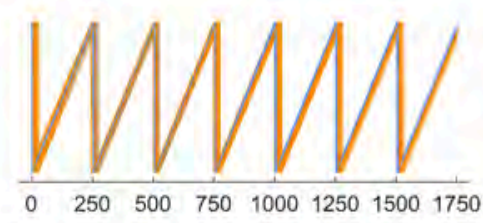

Phase (Hilbert-Transform)
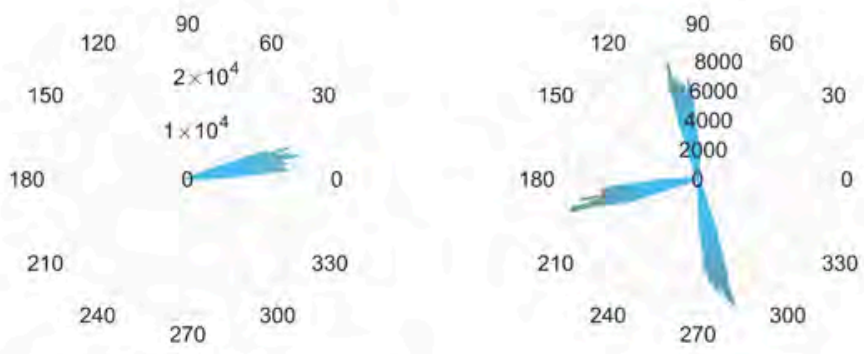

Phase differences (audio - video)
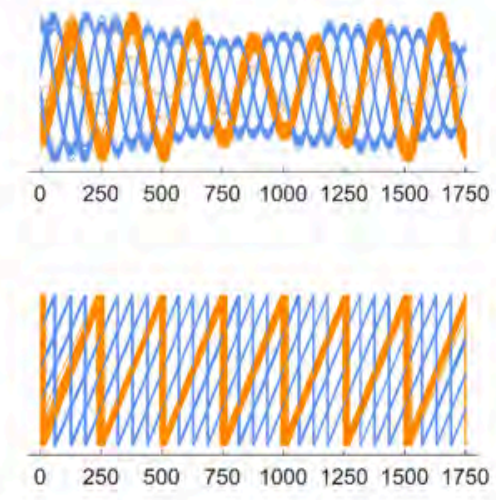

\section{$x^{x+\infty}$}

나

(1)

()

(U)

(u)

$<$

(2) 


\section{A SSVEP}

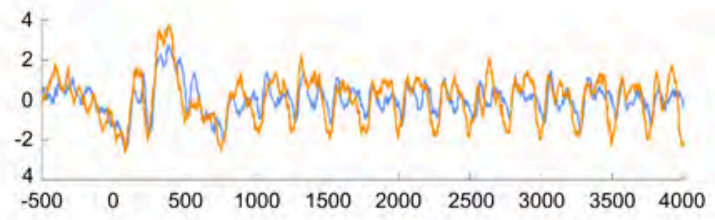
6.
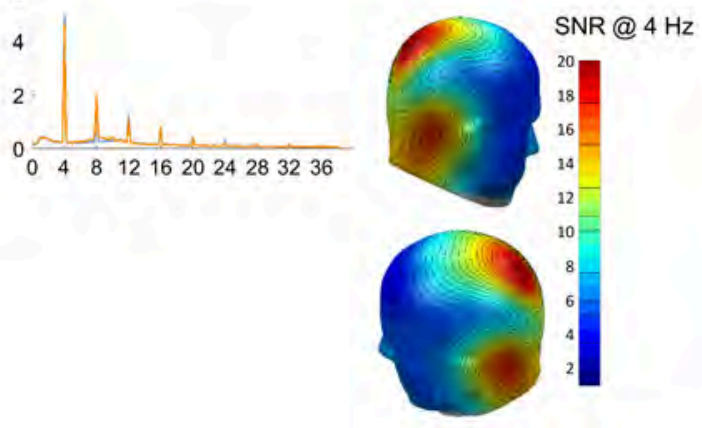

\section{B ASSR}
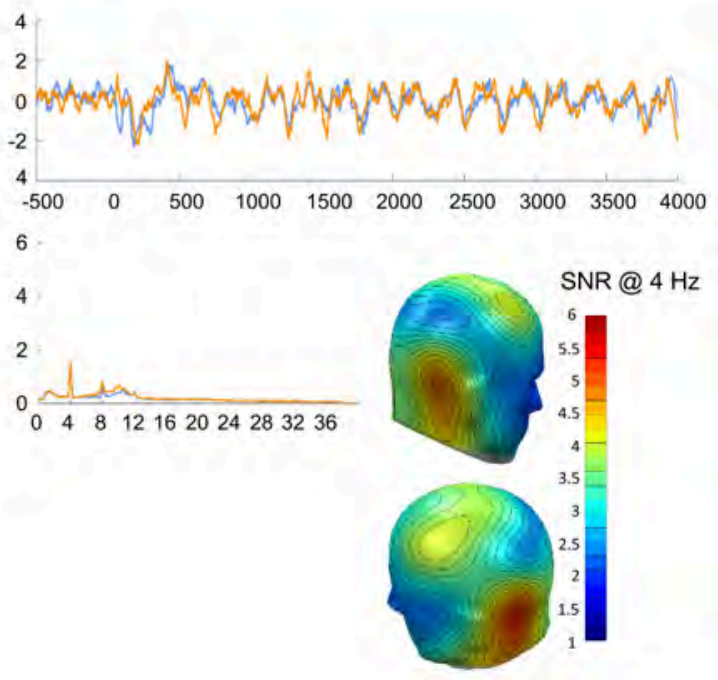
A) Model weights: generalization
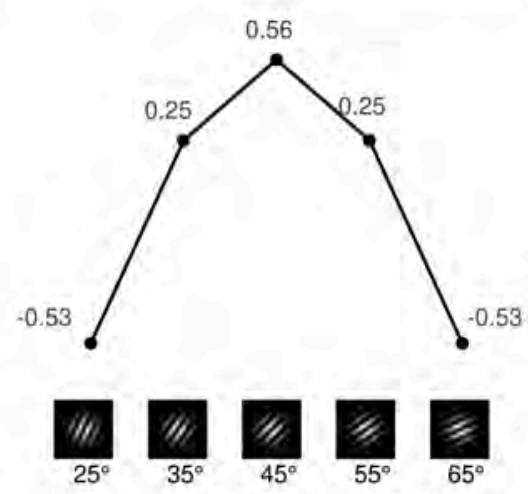

B) Interaction weights: Discrimination
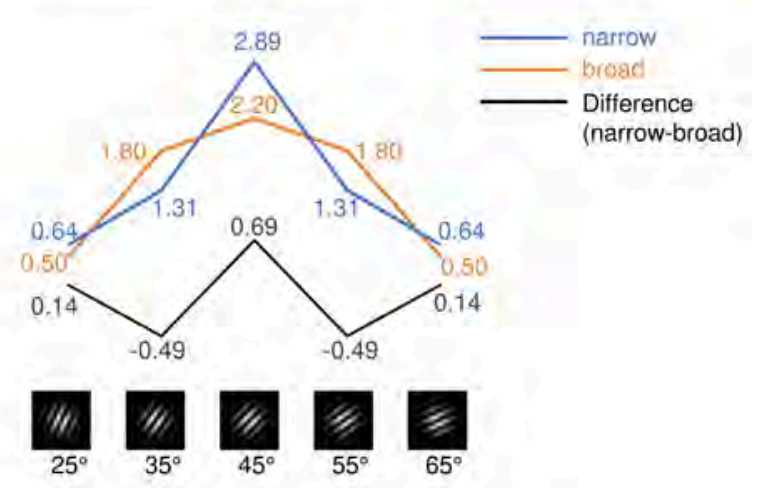


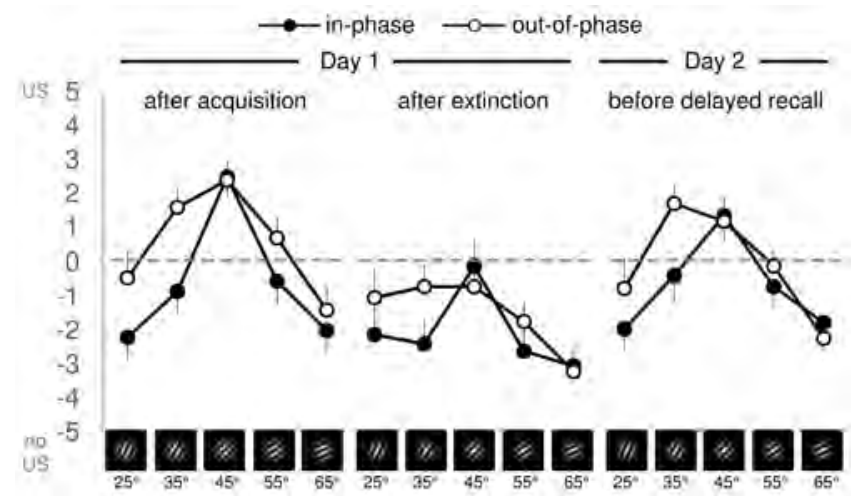


(A) Valence ratings

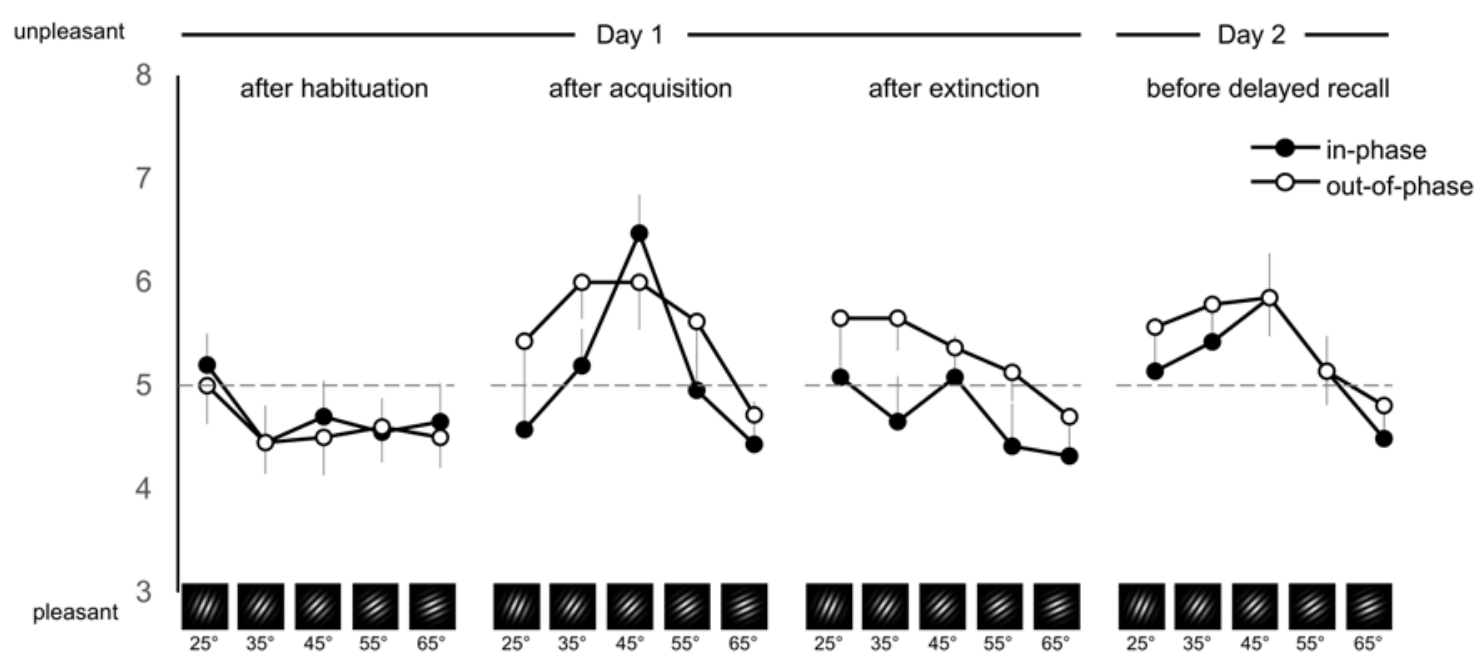

(B) Arousal ratings

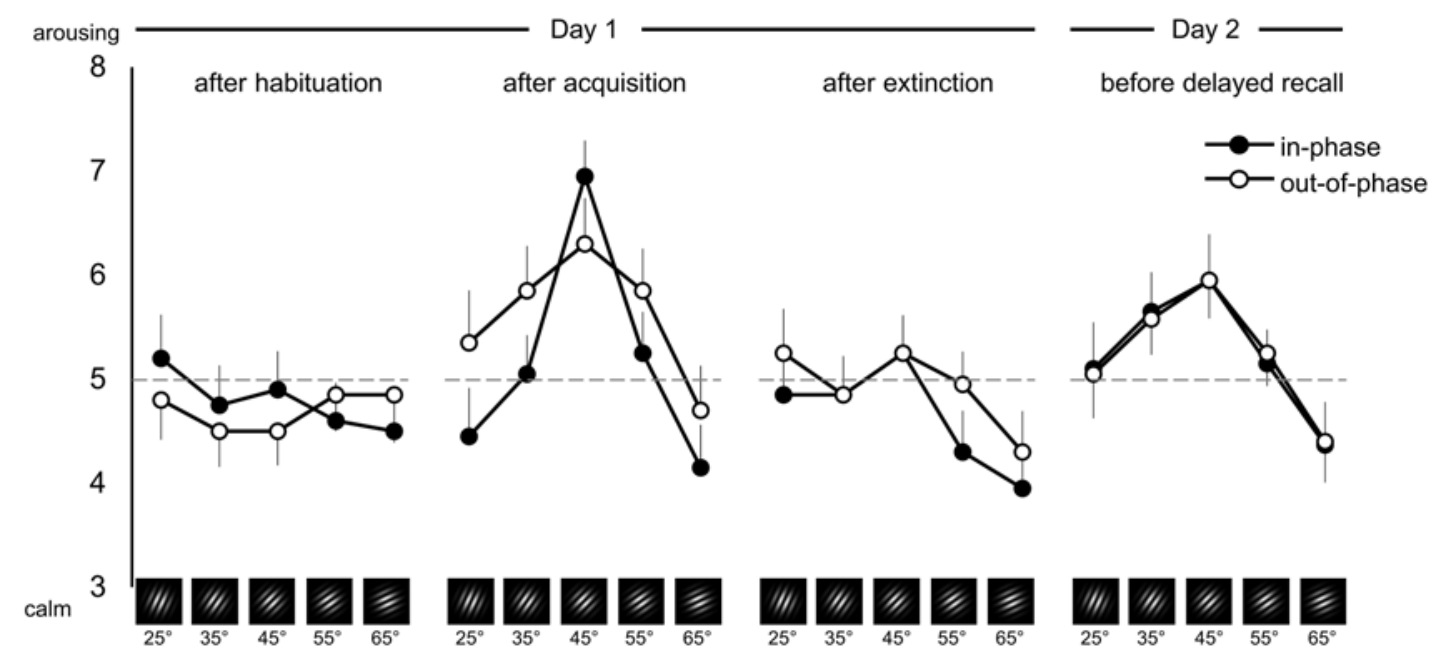


(A) In-phase group: $0^{\circ}$ phase offset
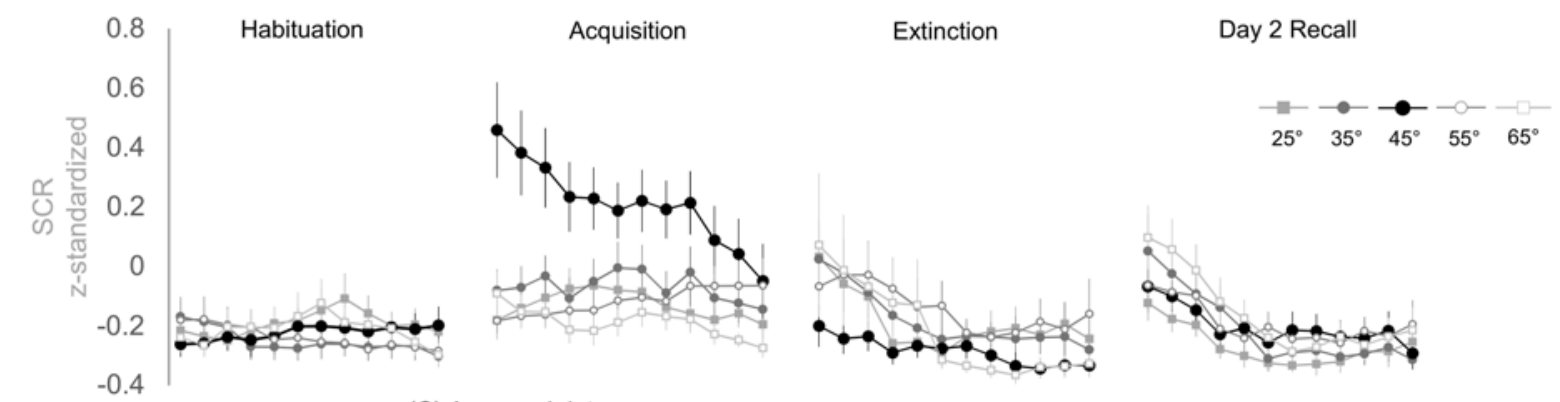

(B) Out-of-phase group: $90^{\circ}$,

$180^{\circ}, 270^{\circ}$ phase offset
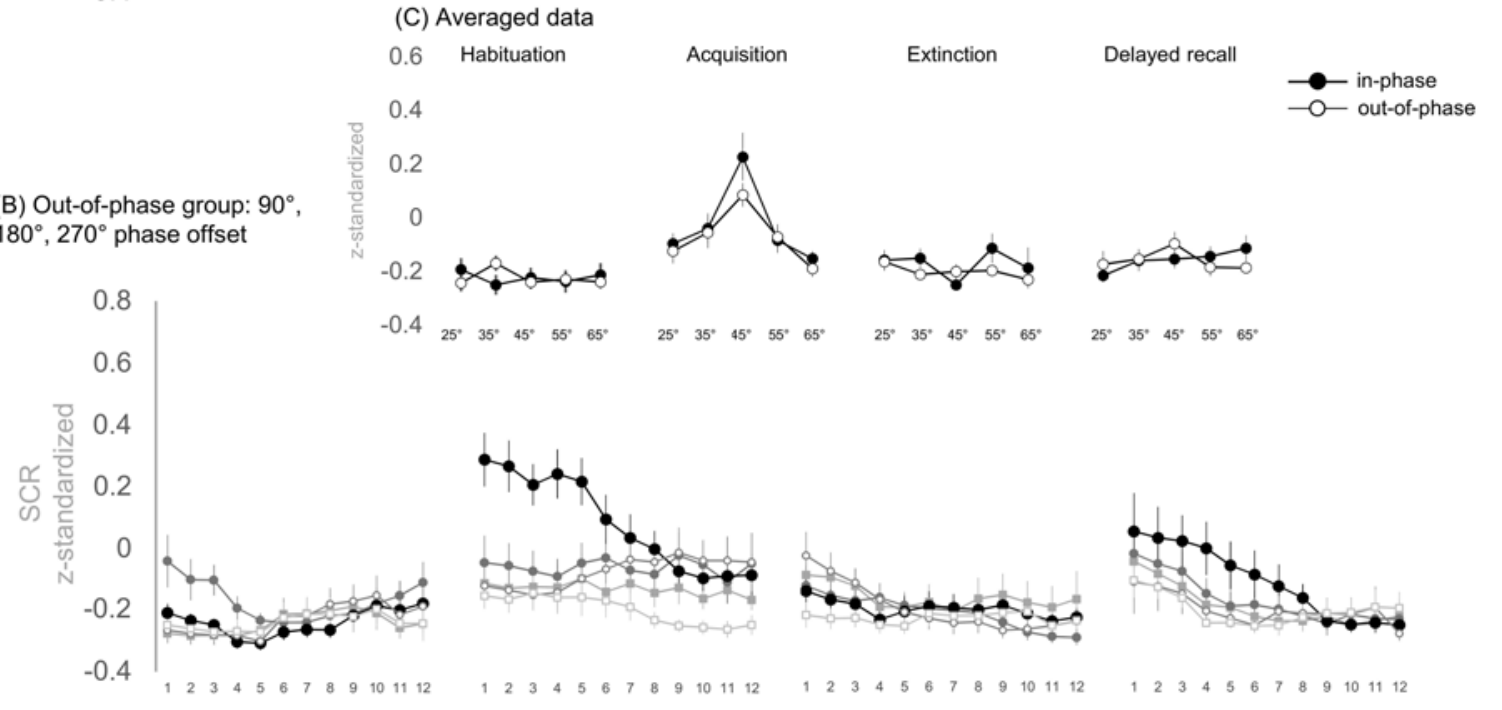
(A) In-phase group: $0^{\circ}$ phase offset

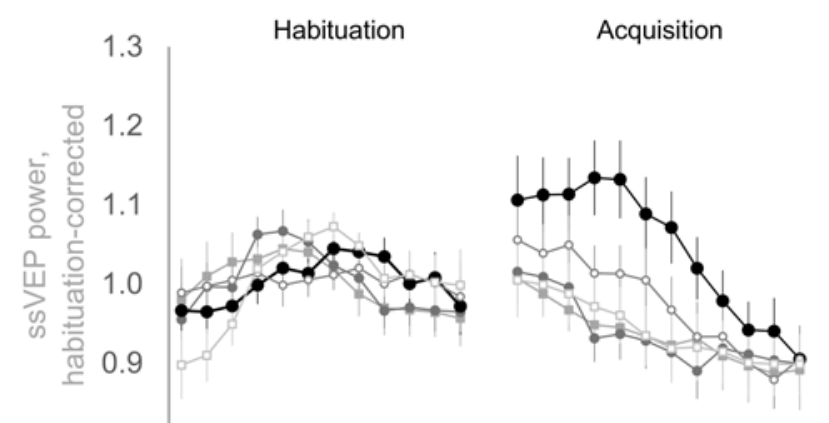

Extinction

C) Averaged data

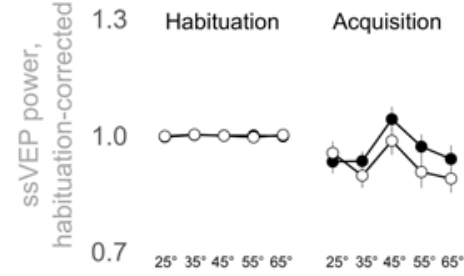

Extinction Delayed Recal

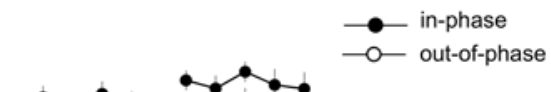

(B) Out-of-phase group: $90^{\circ}$ $180^{\circ}, 270^{\circ}$ phase offset
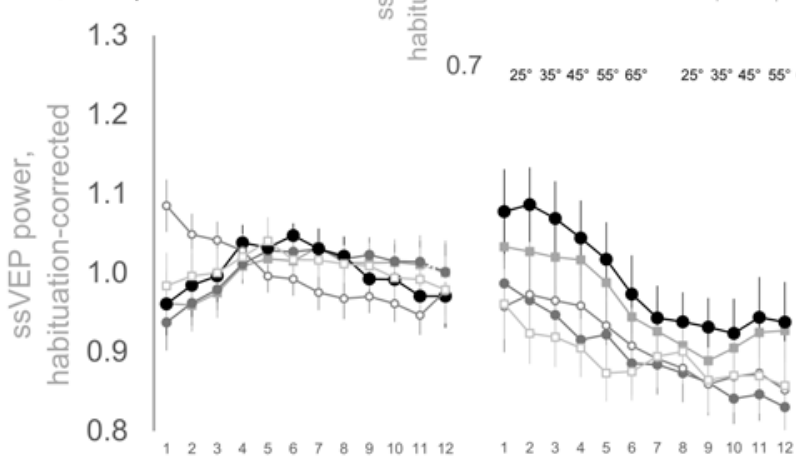
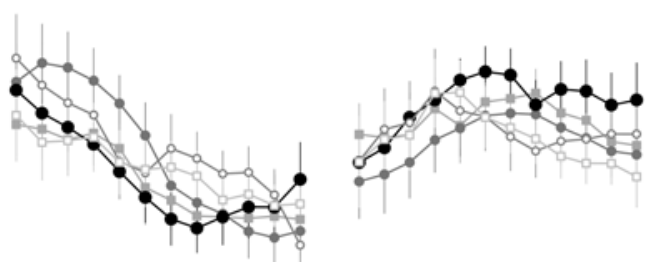
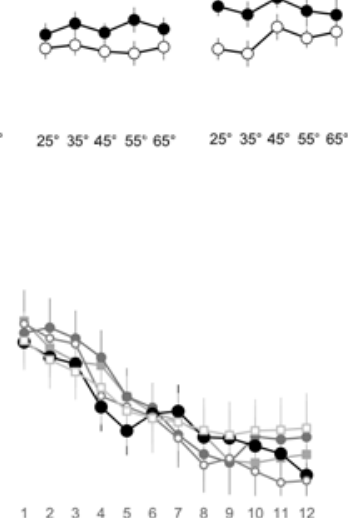

$25^{\circ}-35^{\circ}-45^{\circ}-55^{\circ}-65^{\circ}$

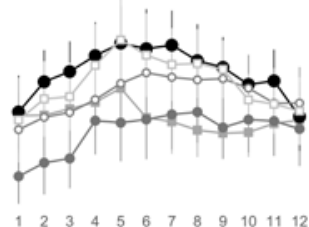

